\title{
Mass Renormalization in the Nelson Model
}

\author{
Fumio Hiroshima ${ }^{1}$ and Susumu Osawa ${ }^{2}$ \\ ${ }^{1}$ Faculty of Mathematics, Kyushu University, Fukuoka 819-0385, Japan \\ ${ }^{2}$ Faculty of Science, Department of Mathematics, Hokkaido University, Sapporo, Hokkaido 060-0810, Japan
}

Correspondence should be addressed to Susumu Osawa; susumu_osawa@kyudai.jp

Received 20 August 2016; Accepted 25 September 2016; Published 6 June 2017

Academic Editor: Rodica D. Costin

Copyright (C) 2017 Fumio Hiroshima and Susumu Osawa. This is an open access article distributed under the Creative Commons Attribution License, which permits unrestricted use, distribution, and reproduction in any medium, provided the original work is properly cited.

\begin{abstract}
The asymptotic behavior of the effective mass $m_{\text {eff }}(\Lambda)$ of the so-called Nelson model in quantum field theory is considered, where $\Lambda$ is an ultraviolet cutoff parameter of the model. Let $m$ be the bare mass of the model. It is shown that for sufficiently small coupling constant $|\alpha|$ of the model, $m_{\mathrm{eff}}(\Lambda) / m$ can be expanded as $m_{\mathrm{eff}}(\Lambda) / m=1+\sum_{n=1}^{\infty} a_{n}(\Lambda) \alpha^{2 n}$. A physical folklore is that $a_{n}(\Lambda)=O\left([\log \Lambda]^{(n-1)}\right)$ as $\Lambda \rightarrow \infty$. It is rigorously shown that $0<\lim _{\Lambda \rightarrow \infty} a_{1}(\Lambda)<C, C_{1} \leq \lim _{\Lambda \rightarrow \infty} a_{2}(\Lambda) / \log \Lambda \leq C_{2}$ with some constants $C, C_{1}$, and $C_{2}$.
\end{abstract}

\section{Introduction and Main Results}

The model considered in this paper is the so-called Nelson model [1], which describes a nonrelativistic nucleon with bare mass $m>0$ interacting with a quantized scalar field with mass $v>0$. The nucleon is governed by a Schrödinger operator. Let us first define the Nelson Hamiltonian. We use relativistic unit and employ the total momentum representation. Then the Hilbert space of states is the boson Fock space over $L^{2}\left(\mathbb{R}^{3}\right)$ which is given by

$$
\mathscr{F}=\bigoplus_{n=0}^{\infty}\left[\bigotimes_{s}^{n} L^{2}\left(\mathbb{R}^{3}\right)\right],
$$

where $\bigotimes_{s}^{n}$ denotes the $n$-fold symmetric tensor product and $\bigotimes_{s}^{0} L^{2}\left(\mathbb{R}^{3}\right)=\mathbb{C}$. Then $\Phi \in \mathscr{F}$ can be written as $\Phi=\left\{\Phi^{(0)}\right.$, $\left.\Phi^{(1)}, \Phi^{(2)}, \ldots\right\}$, where $\Phi^{(n)} \in \bigotimes_{s}^{n} L^{2}\left(\mathbb{R}^{3}\right)$. The Fock vacuum $\Omega \in \mathscr{F}$ is defined by $\Omega=\{1,0,0, \ldots\}$. Let $a(f), f \in L^{2}\left(\mathbb{R}^{3}\right)$, be the annihilation operator and $a(f)^{*}, f \in L^{2}\left(\mathbb{R}^{3}\right)$, the creation operator on $\mathscr{F}$, which are defined by

$$
\begin{aligned}
& D\left(a(f)^{*}\right) \\
& =\left\{\Psi \in \mathscr{F} \mid \sum_{n=0}^{\infty}(n+1)\left\|S_{n+1}\left(f \otimes \Psi^{(n)}\right)\right\|_{\otimes^{n} L^{2}\left(\mathbb{R}^{3}\right)}^{2}<\infty\right\},
\end{aligned}
$$

$$
\begin{aligned}
& \left(a(f)^{*} \Psi\right)^{(0)}=0, \\
& \left(a(f)^{*} \Psi\right)^{(n+1)}=\sqrt{n+1} S_{n+1}\left(f \otimes \Psi^{(n)}\right),
\end{aligned}
$$

and $a(f)=\left(a(f)^{*}\right)^{*}$, where $S_{n}$ is the symmetrizer, $D(X)$ the domain of operator $X$, and $\|\cdot\|_{\mathscr{K}}$ the norm on $\mathscr{K}$. They satisfy canonical commutation relations as follows:

$$
\begin{gathered}
{\left[a(f), a(g)^{*}\right]=(f, g),} \\
{[a(f), a(g)]=0,} \\
{\left[a(f)^{*}, a(g)^{*}\right]=0}
\end{gathered}
$$

on a suitable dense domain, where $[X, Y]=X Y-Y X$ and $(\cdot, \cdot)$ is the inner product on $\mathscr{K}$ (linear in the second variable). Let $T$ be a self-adjoint operator on $L^{2}\left(\mathbb{R}^{3}\right)$. Then we define the self-adjoint operator $d \Gamma(T)$ on $\mathscr{F}$ by $d \Gamma(T)=\bigoplus_{n=0}^{\infty} T^{(n)}$, where

$$
T^{(n)}=\overline{\left(\sum_{j=1}^{n} I \otimes \cdots \otimes I \otimes \overleftrightarrow{T} \overleftrightarrow{\text { th }} \otimes I \otimes \cdots \otimes I\right)[\bar{\otimes} D(T)}
$$


with $T^{(0)}=0$. Here, for a closable operator $T, \bar{T}$ denotes the closure of $T$. The operator $d \Gamma(T)$ is called the second quantization of $T$. The free energy of the scalar field is given by $H_{f}=d \Gamma(\omega)$, where $\omega(k)=\sqrt{|k|^{2}+v^{2}}\left(k=\left(k_{1}, k_{2}, k_{3}\right) \epsilon\right.$ $\left.\mathbb{R}^{3}, v>0\right)$ is considered as a multiplication operator on $L^{2}\left(\mathbb{R}^{3}\right)$. Similarly the momentum of the scalar field is given by $P_{f \mu}=\mathrm{d} \Gamma\left(k_{\mu}\right)(\mu=1,2,3)$. The coupling of the nucleon and a scalar field is mediated through the Segal field operator $\Phi_{s}(g)$ defined by

$$
\Phi_{s}(g)=\frac{1}{\sqrt{2}}\left(a(g)+a(g)^{*}\right)
$$

where $g$ is a cutoff function given by $g(k)=\widehat{\phi}(k) / \sqrt{\omega(k)}$. Here $\widehat{\phi}$ is the form factor with infrared cutoff $\kappa>0$ and ultraviolet cutoff $\Lambda>0$, which are defined by

$$
\widehat{\phi}(k)= \begin{cases}0 & |k|<\kappa, \\ (2 \pi)^{-3 / 2} & \kappa \leq|k| \leq \Lambda, \\ 0 & |k|>\Lambda .\end{cases}
$$

The Nelson Hamiltonian with total momentum $p \in \mathbb{R}^{3}$ is given by a self-adjoint operator on $\mathscr{F}$ as follows:

$$
H(p)=\frac{1}{2 m}\left(p-P_{f}\right)^{2}+H_{f}+\alpha \Phi_{s}(g)
$$

where $\alpha \in \mathbb{R}$ is a coupling constant. Let $E(p, \alpha)$ be the energymomentum relation (the infimum of the spectrum $\sigma(H(p))$ ) defined by

$$
E(p, \alpha)=\inf \sigma(H(p))
$$

Then the effective mass $m_{\text {eff }}=m_{\text {eff }}(\Lambda)$ is defined by

$$
\frac{1}{m_{\mathrm{eff}}}=\frac{1}{3} \Delta_{p} E(p, \alpha) \uparrow_{p=0}
$$

Here $\Delta_{p}$ denotes the three-dimensional Laplacian in the variable $p$. We are concerned with the asymptotic behavior of $m_{\text {eff }}$ as the ultraviolet cutoff goes to infinity. It is however a subtle problem. Removal of the ultraviolet cutoff $\Lambda$ through mass renormalization means finding sequences $\{m\}$ and $\{\Lambda\}$ such that $m \rightarrow 0, \Lambda \rightarrow \infty$, and $m_{\text {eff }}$ converges. Since we can see that $m_{\text {eff }} / m$ is a function of $\Lambda / m$, to achieve this, we want to find constants $0<\gamma<1$ and $0<b_{0}<\infty$ such that

$$
\lim _{\Lambda \rightarrow \infty} \frac{m_{\mathrm{eff}} / m}{(\Lambda / m)^{\gamma}}=b_{0} .
$$

If we succeed in finding constants $\gamma$ and $b_{0}$ such as in (10), scaling the bare mass $m$ as

$$
m=\frac{1}{\Lambda^{\gamma /(1-\gamma)}} M
$$

where $M=\left(m^{*} / b_{0}\right)^{1 /(1-\gamma)}$ with an arbitrary positive constant $m^{*}$, we have

$$
\lim _{\Lambda \rightarrow \infty} m_{\mathrm{eff}}(\Lambda)=m^{*}
$$

The mass renormalization is, however, a subtle problem, and unfortunately, we cannot yet find constants $\gamma$ and $b_{0}$ such as in (10). For that reason we turn to perturbative renormalization, by which we try to guess the proper value of $\gamma$. Main results obtained in this paper are summarized as follows.

Theorem 1. Let $\kappa>0$. Then $m_{\text {eff }}$ is an analytic function of $\alpha^{2}$ and can be expanded in the following power series for sufficiently small $|\alpha|$ :

$$
\frac{m_{e f f}}{m}=1+\sum_{n=1}^{\infty} a_{n}(\Lambda) \alpha^{2 n}
$$

Theorem 2. There exists a strictly positive constant $C$ such that

$$
\lim _{\Lambda \rightarrow \infty} a_{1}(\Lambda)=C
$$

Theorem 3. There exist some constants $C_{1}$ and $C_{2}$ such that

$$
C_{1} \leq \lim _{\Lambda \rightarrow \infty} \frac{a_{2}(\Lambda)}{\log \Lambda} \leq C_{2}
$$

From Theorems 2 and 3, if $D=\lim _{\Lambda \rightarrow \infty} a_{2}(\Lambda) / \log \Lambda>0$, it is suggested that $\gamma=D \alpha^{2} / C$. So, the mass of the Nelson model is renormalizable for sufficiently small $|\alpha|$.

The effective mass and energy-momentum relation have been studied mainly in nonrelativistic electrodynamics. Spohn [2] investigates the upper and lower bound of the effective mass of the polaron model from a functional integral point of view. Hiroshima and Spohn [3] study a perturbative mass renormalization including fourth order in the coupling constant in the case of a spinless electron. Hiroshima and Ito $[4,5]$ study it in the case of an electron with spin $1 / 2$. Bach et al. [6] show that the energy-momentum relation is equal to the infimum of the essential spectrum of the Hamiltonian for $\kappa \geq 0$. Fröhlich and Pizzo [7] investigate energy-momentum relation when infrared cutoff goes to 0 .

\section{Analytic Properties}

In order to investigate the effective mass in a perturbation theory we have to check the analytic properties of $E(p, \alpha)$.

\subsection{Analytic Family in the Sense of Kato}

Lemma 4. $H(p)$ is an analytic family in the sense of Kato.

Proof. We prove $H(p)$ is an analytic family of type (A). We see that

$$
H(p)=H_{0}+\sum_{\mu=1}^{3} p_{\mu} \frac{1}{2 m}\left(p_{\mu}-2 P_{f \mu}\right)+\alpha H_{I},
$$

where $H_{0}=(1 / 2 m) P_{f}^{2}+H_{f}$ and $H_{I}=\Phi_{s}(g)$. Hence all we have to do is to prove the following facts.

(a) $D\left(H_{0}\right) \subset \bigcap_{\mu=1}^{3} D\left(P_{f \mu}\right) \cap D\left(H_{I}\right)$. 
(b) There exist real constants $a_{\mu}, b_{\mu}(\mu=1,2,3), c$, and $d$ such that for any $\Psi \in D\left(H_{0}\right)$

$$
\begin{array}{r}
\left\|\frac{1}{2 m}\left(p_{\mu}-2 P_{f \mu}\right) \Psi\right\|_{\mathscr{F}} \leq a_{\mu}\left\|H_{0} \Psi\right\|_{\mathscr{F}}+b_{\mu}\|\Psi\|_{\mathscr{F}} \\
(\mu=1,2,3),
\end{array}
$$

$\left\|H_{I} \Psi\right\|_{\mathscr{F}} \leq c\left\|H_{0} \Psi\right\|_{\mathscr{F}}+d\|\Psi\|_{\mathscr{F}}$.

We prove (a) at first. Since $\bigcap_{\mu=1}^{3} D\left(P_{f \mu}^{2}\right) \subset D\left(P_{f \mu}\right)$, we have $D\left(H_{0}\right)=\bigcap_{\mu=1}^{3} D\left(P_{f \mu}^{2}\right) \cap D\left(H_{f}\right) \subset \bigcap_{\mu=1}^{3} D\left(P_{f \mu}\right)$. Additionally, since $\left\|\omega^{-1 / 2} g\right\|_{L^{2}\left(\mathbb{R}^{3}\right)}<\infty$, we have $g \in D\left(\omega^{-1 / 2}\right)$. Furthermore, since $\omega$ is a nonnegative and injective self-adjoint operator on $L^{2}\left(\mathbb{R}^{3}\right)$, it follows that $D\left(d \Gamma(\omega)^{1 / 2}\right) \subset D(a(g)) \cap$ $D\left(a(g)^{*}\right)=D\left(H_{I}\right)$. Hence we have $D\left(H_{0}\right) \subset D(d \Gamma(\omega)) \subset$ $D\left(d \Gamma(\omega)^{1 / 2}\right)$. Together with them, (a) is proven. Next we prove (b). Let $\Psi$ be an arbitrary vector in $D\left(H_{0}\right)$. Then we have

$$
\left\|\frac{1}{2 m}\left(p_{\mu}-2 P_{f \mu}\right) \Psi\right\|_{\mathscr{F}} \leq \frac{\left|p_{\mu}\right|}{2 m}\|\Psi\|_{\mathscr{F}}+\frac{1}{m}\left\|P_{f \mu} \Psi\right\|_{\mathscr{F}} .
$$

Since $\left\|P_{f \mu} \Psi\right\|_{\mathscr{F}}^{2} \leq 2 m\left\|H_{0}^{1 / 2} \Psi\right\|_{\mathscr{F}}^{2}$, we have $\left\|H_{0}^{1 / 2} \Psi\right\|_{\mathscr{F}}^{2} \leq \|\left(H_{0}+\right.$ 1) $\Psi \|_{\mathscr{F}}^{2}$. Hence

$$
\begin{aligned}
& \left\|\frac{1}{2 m}\left(p_{\mu}-2 P_{f \mu}\right) \Psi\right\|_{\mathscr{F}} \\
& \quad \leq \sqrt{\frac{2}{m}}\left\|H_{0} \Psi\right\|_{\mathscr{F}}+\left(\frac{\left|p_{\mu}\right|}{2 m}+\sqrt{\frac{2}{m}}\right)\|\Psi\|_{\mathscr{F}} .
\end{aligned}
$$

Since $D\left(H_{0}\right) \subset D\left(d \Gamma(\omega)^{1 / 2}\right)$,

$$
\begin{aligned}
\|a(g) \Psi\|_{\mathscr{F}} \leq & \left\|\omega^{-1 / 2} g\right\|_{L^{2}\left(\mathbb{R}^{3}\right)}\left\|H_{f}^{1 / 2} \Psi\right\|_{\mathscr{F}}, \\
\left\|a(g)^{*} \Psi\right\|_{\mathscr{F}} \leq & \left\|\omega^{-1 / 2} g\right\|_{L^{2}\left(\mathbb{R}^{3}\right)}\left\|H_{f}^{1 / 2} \Psi\right\|_{\mathscr{F}} \\
& +\|g\|_{L^{2}\left(\mathbb{R}^{3}\right)}\|\Psi\|_{\mathscr{F}}
\end{aligned}
$$

hold. Hence

$$
\begin{aligned}
\left\|H_{I} \Psi\right\|_{\mathscr{F}} \leq & \sqrt{2}\left\|\omega^{-1 / 2} g\right\|_{L^{2}\left(\mathbb{R}^{3}\right)}\left\|H_{f}^{1 / 2} \Psi\right\|_{\mathscr{F}} \\
& +\frac{1}{\sqrt{2}}\|g\|_{L^{2}\left(\mathbb{R}^{3}\right)}\|\Psi\|_{\mathscr{F}} .
\end{aligned}
$$

From triangle inequality, we have $\left\|H_{f}^{1 / 2} \Psi\right\|_{\mathscr{F}} \leq\left\|H_{f} \Psi\right\|_{\mathscr{F}}+$ $\|\Psi\|_{\mathscr{F}}$. In addition,

$$
\begin{aligned}
\left\|H_{0} \Psi\right\|_{\mathscr{F}}^{2}-\left\|H_{f} \Psi\right\|_{\mathscr{F}}^{2}= & \left\|\frac{1}{2 m} P_{f}^{2} \Psi\right\|_{\mathscr{F}}^{2} \\
& +\frac{1}{m} \mathfrak{R}\left(P_{f}^{2} \Psi, H_{f} \Psi\right) .
\end{aligned}
$$

Since $P_{f}^{2}$ and $H_{f}$ are strongly commutative and nonnegative self-adjoint operators on $\mathscr{F},\left(P_{f}^{2} \Psi, H_{f} \Psi\right) \geq 0$ holds. Hence $\left\|H_{f} \Psi\right\|_{\mathscr{F}} \leq\left\|H_{0} \Psi\right\|_{\mathscr{F}}$. Then we have

$$
\begin{aligned}
& \left\|H_{I} \Psi\right\|_{\mathscr{F}} \\
& \leq \sqrt{2}\left\|\omega^{-1 / 2} g\right\|_{L^{2}\left(\mathbb{R}^{3}\right)}\left\|H_{0} \Psi\right\|_{\mathscr{F}} \\
& \quad+\left(\sqrt{2}\left\|\omega^{-1 / 2} g\right\|_{L^{2}\left(\mathbb{R}^{3}\right)}+\frac{1}{\sqrt{2}}\|g\|_{L^{2}\left(\mathbb{R}^{3}\right)}\right)\|\Psi\|_{\mathscr{F}} .
\end{aligned}
$$

From (19) and (23), (b) is proven. Hence $H(p)$ is an analytic family of type (A). Since every analytic family of type (A) is an analytic family of in the sense of Kato, it is an analytic family in the sense of Kato.

We denote the ground state of $H(p)$ by $\psi_{g}(p)$.

Lemma 5. (1) $E(p, \alpha)$ is analytic in $p$ and $\alpha$ if $|p|$ and $|\alpha|$ are sufficiently small. $(2) \psi_{g}(p)$ is strongly analytic in $p$ and $\alpha$ if $|p|$ and $|\alpha|$ are sufficiently small.

Proof. From [8, Theorem XII.9], (1) follows, and from [8, Theorem XII.8], (2) follows.

2.2. Formula. In this section we expand $m / m_{\text {eff }}$ with respect to $\alpha$.

Lemma 6. The ratio $\mathrm{m} / \mathrm{m}_{\text {eff }}$ can be expressed as

$$
\frac{m}{m_{\text {eff }}}=1-\frac{2}{3} \sum_{\mu=1}^{3} \frac{\left(P_{f \mu} \psi_{g}(0), \psi_{g_{\mu}}^{\prime}(0)\right)}{\left(\psi_{g}(0), \psi_{g}(0)\right)}
$$

where $\psi_{g_{\mu}}^{\prime}(0)=s-\partial p_{\mu} \psi_{g}(p) \Gamma_{p=0}$.

Proof. Since $E(p, \alpha)$ is symmetry, $E(p, \alpha)=E(-p, \alpha)$, we have

$$
\partial p_{\mu} E(p, \alpha) \uparrow_{p=0}=0, \quad \mu=1,2,3 .
$$

Since $H(p) \psi_{g}(p)=E(p, \alpha) \psi_{g}(p)$, for any $\Psi \in D(H(p))$,

$$
\left(H(p) \Psi, \psi_{g}(p)\right)=E(p, \alpha)\left(\Psi, \psi_{g}(p)\right)
$$

holds. Taking a derivative with respect to $p_{\mu}$ on both sides above, we have

$$
\begin{aligned}
& \left(H_{\mu}^{\prime}(p) \Psi, \psi_{g}(p)\right) \\
& \quad+\left(H(p) \Psi, \psi_{g_{\mu}}^{\prime}(p)\right)=E_{\mu}^{\prime}(p, \alpha)\left(\Psi, \psi_{g}(p)\right) \\
& \quad+E(p, \alpha)\left(\Psi, \psi_{g_{\mu}}^{\prime}(p)\right), \\
& \left(H_{\mu}^{\prime \prime}(p) \Psi, \psi_{g}(p)\right)+2\left(H_{\mu}^{\prime}(p) \Psi, \psi_{g_{\mu}}^{\prime}(p)\right) \\
& \quad+\left(H(p) \Psi, \psi_{g_{\mu}}^{\prime \prime}(p)\right)=E_{\mu}^{\prime \prime}(p, \alpha)\left(\Psi, \psi_{g}(p)\right) \\
& \quad+2 E_{\mu}^{\prime}(p, \alpha)\left(\Psi, \psi_{g_{\mu}}^{\prime}(p)\right)+E(p, \alpha)\left(\Psi, \psi_{g_{\mu}}^{\prime \prime}(p)\right) .
\end{aligned}
$$


Here ' denotes the derivative or strong derivative with respect to $p_{\mu}$, and $H_{\mu}^{\prime}(p)=(1 / m)\left(p_{\mu}-P_{f \mu}\right), H_{\mu}^{\prime \prime}(p)=1 / m$. Setting $\Psi=\psi_{g}(0)$ and $p=0$, we have

$$
\begin{aligned}
& E_{\mu}^{\prime \prime}(0, \alpha) \\
& \quad=\frac{1}{m} \frac{\left(\psi_{g}(0), \psi_{g}(0)\right)-2\left(P_{f \mu} \psi_{g}(0), \psi_{g \mu}^{\prime}(0)\right)}{\left(\psi_{g}(0), \psi_{g}(0)\right)} .
\end{aligned}
$$

This expression and the definition of the effective mass prove the lemma.

2.3. Perturbative Expansions. We define operators $A^{+}$and $A^{-}$ by $A^{+}=(1 / \sqrt{2}) a(g)^{*}$ and $A^{-}=(1 / \sqrt{2}) a(g)$. Then $H_{I}=$ $A^{+}+A^{-}$. Moreover, let $\mathscr{F}^{(n)}=\bigotimes_{s}^{n} L^{2}\left(\mathbb{R}^{3}\right)$ and

$$
\psi_{g}(0)=\sum_{n=0}^{\infty} \frac{\alpha^{n}}{n !} \varphi_{n}
$$

Since $E(p, \alpha)$ is symmetry $E(p,-\alpha)=E(p, \alpha)$, we have

$$
E(0, \alpha)=\sum_{n=0}^{\infty} \frac{\alpha^{2 n}}{(2 n) !} E_{2 n}
$$

Since ker $H_{0} \neq\{0\}, H_{0}$ is not injective. However, we define the operator $1 / H_{0}$ (for notational simplicity we write $1 / H_{0}$ for $H_{0}^{-1}$ in what follows) on $\mathscr{F}$ as follows.

$$
\begin{aligned}
& D\left(\frac{1}{H_{0}}\right) \\
& \quad=\left\{\Psi=\bigoplus_{n=0}^{\infty} \Psi^{(n)} \in \mathscr{F} \mid \sum_{n=1}^{\infty}\left\|\beta^{n} \Psi^{(n)}\right\|^{2}<\infty\right\}, \\
& \left(\frac{1}{H_{0}} \Psi\right)^{(0)}=0, \\
& \left(\frac{1}{H_{0}} \Psi\right)^{(n)}\left(k_{1}, \ldots, k_{n}\right) \\
& \quad=\beta^{n}\left(k_{1}, \ldots, k_{n}\right) \Psi^{(n)}\left(k_{1}, \ldots, k_{n}\right) \quad(n \geq 1) .
\end{aligned}
$$

Here

$$
\begin{aligned}
\beta^{n} & =\beta^{n}\left(k_{1}, \ldots, k_{n}\right) \\
& =\frac{1}{(1 / 2 m)\left|k_{1}+\cdots+k_{n}\right|^{2}+\sum_{i=1}^{n} \omega\left(k_{i}\right)} .
\end{aligned}
$$

We define the subspace $\mathscr{F}_{\text {fin }}$ of $\mathscr{F}$ as $\mathscr{F}_{\text {fin }}=\left\{\left\{\Psi^{(n)}\right\}_{n=0}^{\infty} \in \mathscr{F} \mid\right.$ $\Psi^{(l)}=0$ for $l \geq q$ with some $\left.q\right\}$.

Lemma 7. It holds that $\mathscr{F}_{\text {fin }} \subset D\left(1 / H_{0}\right)$.

Proof. Let $\Psi \in \mathscr{F}_{\text {fin }}$. Then $\left\|\left(1 / H_{0}\right) \Psi\right\|^{2}=\sum_{n=1}^{\infty}\left\|\left(\left(1 / H_{0}\right) \Psi\right)^{(n)}\right\|^{2}<$ $\infty$. Hence the lemma follows.
Lemma 8. Let $\psi_{g}(0)=\sum_{n=0}^{\infty}\left(\alpha^{n} / n !\right) \varphi_{n}$. Then $\varphi_{0}=\Omega, \varphi_{1}=$ $-\left(1 / H_{0}\right) H_{I} \Omega$, and the recurrence formulas

$$
\begin{aligned}
& \varphi_{2 l}=\frac{1}{H_{0}}\left\{-2 l H_{I} \varphi_{2 l-1}+\sum_{j=1}^{l}\left(\begin{array}{l}
2 l \\
2 j
\end{array}\right) E_{2 j} \varphi_{2 l-2 j}\right\} \\
&(l \geq 1),
\end{aligned}
$$

$\varphi_{2 l+1}$

$$
=\frac{1}{H_{0}}\left\{-(2 l+1) H_{I} \varphi_{2 l}+\sum_{j=1}^{l}\left(\begin{array}{c}
2 l+1 \\
2 j
\end{array}\right) E_{2 j} \varphi_{2 l+1-2 j}\right\}
$$

follow, with

$$
\begin{gathered}
\varphi_{2 l} \in \mathscr{F}^{(2)} \oplus \mathscr{F}^{(4)} \oplus \cdots \oplus \mathscr{F}^{(2 l)} \quad(l \geq 1), \\
\varphi_{2 l+1} \in \mathscr{F}^{(1)} \oplus \mathscr{F}^{(3)} \oplus \cdots \oplus \mathscr{F}^{(2 l+1)} \quad(l \geq 0),
\end{gathered}
$$

and $E_{2 l}$ is given by

$$
E_{2 l}=2 l\left(\Omega, H_{I} \varphi_{2 l-1}\right) \quad(l \geq 1) .
$$

Proof. We have $E(0,0)=E_{0}$ by substituting $\alpha=0$ in (30). Since $E(0,0)$ is the ground state energy of $H_{0}, E(0,0)=0$. Hence $E_{0}=0$. Since $\varphi_{0}$ is the ground state of $H_{0}, \varphi_{0}$ can be $\Omega$. We can find that $\left(\varphi_{n}, \Omega\right)=\delta_{0 n}$ for $n=0,1, \ldots$ holds in the same way as [3]. From now we set $H=H(p)$, $\psi_{g}=\psi_{g}(0), E=E(p, \alpha)$, and ' means (strong)derivative with respect to $\alpha$.

$$
\left(H \Psi, \psi_{g}\right)=E\left(\Psi, \psi_{g}\right)
$$

holds for $\Psi \in D(H)$. Differentiating (37) with respect to $\alpha$, we have

$$
\left(H_{I} \Psi, \psi_{g}\right)+\left(H \Psi, \psi_{g}^{\prime}\right)=E^{\prime}\left(\Psi, \psi_{g}\right)+E\left(\Psi, \psi_{g}^{\prime}\right) .
$$

Hence $\psi_{g}^{\prime} \in D(H)$ and we have

$$
H_{I} \psi_{g}+H \psi_{g}^{\prime}=E^{\prime} \psi_{g}+E \psi_{g}^{\prime} .
$$

Substituting $p=0$ and $\alpha=0$ into (39) and taking into account $\left(\varphi_{n}, \Omega\right)=\delta_{0 n}$, we have $\varphi_{1}=-\left(1 / H_{0}\right) H_{I} \Omega$. Differentiating (37) $n$ times with respect to $\alpha$, we also have

$$
\begin{array}{r}
\left(H \Psi, \psi_{g}^{(n)}\right)+n\left(\Psi, H_{I} \psi_{g}^{(n-1)}\right) \\
=\sum_{j=1}^{n}\left(\begin{array}{l}
n \\
j
\end{array}\right) E^{(j)}\left(\Psi, \psi_{g}^{(n-j)}\right) .
\end{array}
$$

By the induction on $n$, we have $\psi_{g}^{(n)} \in D(H)$ and

$$
H \psi_{g}^{(n)}+n H_{I} \psi_{g}^{(n-1)}=\sum_{j=1}^{n}\left(\begin{array}{l}
n \\
j
\end{array}\right) E^{(j)} \psi_{g}^{(n-j)} .
$$


Substituting $p=0$ and $\alpha=0$ into both sides above, we have

$$
\begin{gathered}
H_{0} \varphi_{2 l}+2 l H_{I} \varphi_{2 l-1}=\sum_{j=1}^{l}\left(\begin{array}{c}
2 l \\
2 j
\end{array}\right) E_{2 j} \varphi_{2 l-2 j} \\
\quad(l \geq 1), \\
H_{0} \varphi_{2 l+1}+(2 l+1) H_{I} \varphi_{2 l}=\sum_{j=1}^{l}\left(\begin{array}{c}
2 l+1 \\
2 j
\end{array}\right) E_{2 j} \varphi_{2 l+1-2 j}
\end{gathered}
$$

From now on, we shall prove

$$
\begin{aligned}
\varphi_{n}^{(i)} & =0, \quad(i>n, \quad i=0), \\
\varphi_{2 l}^{(2 i+1)} & =0, \quad(l \geq 1, \quad 0 \leq i \leq l-1), \\
\operatorname{supp}_{k \in \mathbb{R}^{3 \cdot 2 i}} \varphi_{2 l}^{(2 i)}(k) & =S_{2 i} \text { or } \emptyset, \quad(l \geq 1, \quad 1 \leq i \leq l), \\
\varphi_{2 l+1}^{(2 i)} & =0, \quad(l \geq 0, \quad 0 \leq i \leq l), \\
\operatorname{supp}_{k \in \mathbb{R}^{3(2 i+1)}} \varphi_{2 l+1}^{(2 i+1)}(k) & =S_{2 i+1} \quad \text { or } \emptyset, \quad(l \geq 0,0 \leq i \leq l),
\end{aligned}
$$

where we set $\varphi_{n}=\left\{\varphi_{n}^{(i)}\right\}_{i=0}^{\infty}$ by induction for $n \geq 1$, and

$$
S_{i}=\left\{\left(k_{1}, \ldots, k_{i}\right) \in \mathbb{R}^{3 i}|\kappa \leq| k_{1}|\leq \Lambda, \ldots, \kappa \leq| k_{i} \mid \leq \Lambda\right\} .
$$

Since $\varphi_{1}=-\left(1 / H_{0}\right) H_{I} \Omega \in \mathscr{F}^{(1)}, \varphi_{1}^{(i)}=0, i>1, i=0$. Moreover, since

$$
\varphi_{1}^{(1)}\left(k_{1}\right)=-\frac{1}{\sqrt{2}(2 \pi)^{3 / 2} \sqrt{\omega\left(k_{1}\right)} E\left(k_{1}\right)} \chi_{[\kappa, \Lambda]}\left(\left|k_{1}\right|\right),
$$

we have $\operatorname{supp}_{k_{1} \in \mathbb{R}^{3}} \varphi_{1}^{(1)}\left(k_{1}\right)=S_{1}$, where $E(k)=|k|^{2} / 2 m+\omega(k)$. Assume that the assumption of the induction holds when $n \leq$ $2 l+1,(l \geq 0)$. Then

$$
\begin{aligned}
& H_{0} \varphi_{2 l+2}+(2 l+2) H_{I} \varphi_{2 l+1} \\
& =\sum_{j=1}^{l+1}\left(\begin{array}{c}
2 l+2 \\
2 j
\end{array}\right) E_{2 j} \varphi_{2 l+2-2 j} .
\end{aligned}
$$

It is derived that $\varphi_{2 l+2}^{(i)}=0, i>2 l+2, i=0$, by $\left(\varphi_{n}, \Omega\right)=\delta_{0 n}$ and (46). By the assumption of the induction, $\left(H_{I} \varphi_{2 l+1}\right)^{(2 i+1)}=0,0 \leq i \leq l$, holds. When $1 \leq q \leq l$, it holds that

$$
\begin{aligned}
& \left(H_{0} \varphi_{2 l+2}\right)^{(2 q)}=-2(l+1)\left(H_{I} \varphi_{2 l+1}\right)^{(2 q)} \\
& +\sum_{j=1}^{l+1}\left(\begin{array}{c}
2 l+2 \\
2 j
\end{array}\right) E_{2 j} \varphi_{2 l+2-2 j}^{(2 q)}=-\sqrt{2}(l+1)\left\{\frac{1}{\sqrt{2 q}}\right. \\
& \quad \cdot \sum_{i=1}^{2 q} \frac{1}{(2 \pi)^{3 / 2}} \frac{\chi_{[\kappa, \Lambda]}\left(\left|k_{i}\right|\right)}{\sqrt{2} \sqrt{\omega\left(k_{i}\right)}} \varphi_{2 l+1}^{(2 q-1)}\left(k_{1}, \ldots, \widehat{k}_{i}, \ldots, k_{2 q}\right)
\end{aligned}
$$

$$
\begin{aligned}
& +\sqrt{2 q+1} \int \frac{1}{(2 \pi)^{3 / 2}} \frac{\chi_{[\kappa, \Lambda]}(|k|)}{\sqrt{2} \sqrt{\omega(k)}} \varphi_{2 l+1}^{(2 q+1)}\left(k, k_{1}, \ldots,\right. \\
& \left.\left.k_{2 q}\right) d k\right\}+\sum_{j=1}^{l+1}\left(\begin{array}{c}
2 l+2 \\
2 j
\end{array}\right) E_{2 j} \varphi_{2 l+1-2 j}^{(2 q)}
\end{aligned}
$$

where $\widehat{k}_{i}$ means that $k_{i}$ is omitted. By the assumption of the induction, the supports of the functions

$$
\begin{aligned}
& \frac{1}{(2 \pi)^{3 / 2}} \frac{\chi_{[\kappa, \Lambda]}\left(\left|k_{i}\right|\right)}{\sqrt{2} \sqrt{\omega\left(k_{i}\right)}} \varphi_{2 l+1}^{(2 q-1)}\left(k_{1}, \ldots, \widehat{k}_{i}, \ldots, k_{2 q}\right), \\
& \int \frac{1}{(2 \pi)^{3 / 2}} \frac{\chi_{[\kappa, \Lambda]}(|k|)}{\sqrt{2} \sqrt{\omega(k)}} \varphi_{2 l+1}^{(2 q+1)}\left(k, k_{1}, \ldots, k_{2 q}\right) d k
\end{aligned}
$$

and $\varphi_{2 l+1-2 j}^{(2 q)}$ are $S_{2 q}$ or $\emptyset$. Furthermore,

$$
\begin{aligned}
& \left(H_{0} \varphi_{2 l+2}\right)^{(2 l+2)}\left(k_{1}, \ldots, k_{2 l+2}\right)=-2(l+1) \\
& \cdot\left(A^{+} \varphi_{2 l+1}\right)^{(2 l+2)}\left(k_{1}, \ldots, k_{2 l+2}\right) \\
& =-\sqrt{2(l+1)} \sum_{i=1}^{2 l+2} \frac{1}{(2 \pi)^{3 / 2}} \frac{\chi_{[\kappa, \Lambda]}\left(\left|k_{i}\right|\right)}{\sqrt{2} \sqrt{\omega\left(k_{i}\right)}} \\
& \cdot \varphi_{2 l+1}^{(2 l+1)}\left(k_{1}, \ldots, \widehat{k}_{i}, \ldots, k_{2 l+2}\right)
\end{aligned}
$$

holds. By the assumption of the induction, the support of the right hand side is $S_{2 l+2}$ or $\emptyset$. Hence we have $\operatorname{supp}_{k \in \mathbb{R}^{3 \cdot 2 i}} \varphi_{2 l+2}^{(2 i)}(k)=S_{2 i}$ or $\emptyset, 1 \leq i \leq l+1$. We can prove $\varphi_{2 l+3}^{(i)}=0, i>2 l+3, i=0, \varphi_{2 l+3}^{(2 i)}=0,1 \leq i \leq l+1$, and $\operatorname{supp}_{k \in \mathbb{R}^{3(2 i+1)}} \varphi_{2 l+3}^{(2 i+1)}(k)=S_{2 i+1}$ or $\emptyset, 0 \leq i \leq l+1$, in a similar way. From the discussion so far, we have

$$
-2 l H_{I} \varphi_{2 l-1}+\sum_{j=1}^{l}\left(\begin{array}{l}
2 l \\
2 j
\end{array}\right) E_{2 j} \varphi_{2 l-2 j} \in \mathscr{F}_{\text {fin }}
$$

$(l \geq 1)$,

$$
-(2 l+1) H_{I} \varphi_{2 l}+\sum_{j=1}^{l}\left(\begin{array}{c}
2 l+1 \\
2 j
\end{array}\right) E_{2 j} \varphi_{2 l+1-2 j} \in \mathscr{F}_{\text {fin }}
$$

Hence we have

$$
\begin{aligned}
& \varphi_{2 l}= \frac{1}{H_{0}}\left\{-2 l H_{I} \varphi_{2 l-1}+\sum_{j=1}^{l}\left(\begin{array}{l}
2 l \\
2 j
\end{array}\right) E_{2 j} \varphi_{2 l-2 j}\right\}+b_{2 l} \Omega \\
&(l \geq 1), \\
& \varphi_{2 l+1} \\
&=\frac{1}{H_{0}}\left\{\begin{array}{c}
\left.-(2 l+1) H_{I} \varphi_{l}+\sum_{j=1}^{l}\left(\begin{array}{c}
2 l+1 \\
2 j
\end{array}\right) E_{2 j} \varphi_{2 l+1-2 j}\right\} \\
+b_{2 l+1} \Omega \quad(l \geq 0),
\end{array}\right.
\end{aligned}
$$


where $b_{2 l}$ and $b_{2 l+1}$ are some constants. Since $\left(\varphi_{2 l}, \Omega\right)=0, l \geq$ 1 , and $\left(\varphi_{2 l+1}, \Omega\right)=0, l \geq 0, b_{2 l}=b_{2 l+1}=0$. Hence (33) and (34) are proven. By the discussion so far, (35) are also proven. We can derive (36) by (33) and $\left(\varphi_{n}, \Omega\right)=\delta_{0 n}$.

\section{Main Theorems}

For notational simplicity we set $\widehat{\phi}_{j}=\widehat{\phi}\left(k_{j}\right)$ and $\omega_{j}=\omega\left(k_{j}\right)$ for $k_{j} \in \mathbb{R}^{3}, j=1,2$. Let

$$
\begin{aligned}
E_{j} & =\frac{\left|k_{j}\right|^{2}}{2 m}+\omega_{j}, \quad j=1,2, \\
E_{12} & =\frac{\left|k_{1}+k_{2}\right|^{2}}{2 m}+\omega_{1}+\omega_{2}, \\
\omega(r) & =\sqrt{r^{2}+v^{2}}, \\
F(r) & =\frac{r^{2}}{2 m}+\omega(r) .
\end{aligned}
$$

Theorem 9. Let $\kappa>0$. Then $m_{\text {eff }}$ is an analytic function of $\alpha^{2}$ and can be expanded in the following power series for sufficiently small $|\alpha|$ :

$$
\frac{m_{e f f}}{m}=1+\sum_{n=1}^{\infty} a_{n}(\Lambda) \alpha^{2 n}
$$

Proof. By the power series (29), we have

$$
\begin{aligned}
\left(\psi_{g}, \psi_{g}\right) & =\left(\sum_{n=0}^{\infty} \frac{\alpha^{n}}{n !} \varphi_{n}, \sum_{m=0}^{\infty} \frac{\alpha^{m}}{m !} \varphi_{m}\right) \\
& =\sum_{n=0}^{\infty} \sum_{m=0}^{\infty} \frac{\alpha^{n+m}}{n ! m !}\left(\varphi_{n}, \varphi_{m}\right) .
\end{aligned}
$$

By Lemma $8,\left(\varphi_{n}, \varphi_{m}\right) \neq 0$ if and only if both $n$ and $m$ are even or odd. Then we have

$$
\left(\psi_{g}, \psi_{g}\right)=1+\sum_{n=1}^{\infty} b_{n}(\Lambda) \alpha^{2 n}
$$

From the fact that both $m_{\text {eff }}^{-1}$ and $\left(\psi_{g}, \psi_{g}\right)$ are analytic functions of $\alpha^{2}$ and Lemma 6, we have the following power series:

$$
-\frac{2}{3} \sum_{\mu=1}^{3}\left(P_{f \mu} \psi_{g}, \psi_{g_{\mu}}^{\prime}(0)\right)=\sum_{n=0}^{\infty} c_{n}(\Lambda) \alpha^{2 n} .
$$

Since $\psi_{g_{\mu}}^{\prime}(0)$ is an analytic function of $\alpha$, we can write

$$
\psi_{g_{\mu}}^{\prime}(0)=\sum_{n=0}^{\infty} \frac{\alpha^{n}}{n !} \Phi_{n}^{\mu}
$$

We note that

$$
\begin{aligned}
c_{0}(\Lambda) & =-\frac{2}{3} \sum_{\mu=1}^{3}\left(P_{f \mu} \varphi_{0}, \Phi_{0}^{\mu}\right) \\
& =-\frac{2}{3} \sum_{\mu=1}^{3}\left(d \Gamma\left(k_{\mu}\right) \varphi_{0}, \Phi_{0}^{\mu}\right)=0 .
\end{aligned}
$$

Hence if $|\alpha|$ is sufficiently small, then we have the following power series:

$$
\begin{aligned}
\frac{m_{\mathrm{eff}}}{m} & =\frac{\left(\psi_{g}, \psi_{g}\right)}{\left(\psi_{g}, \psi_{g}\right)-(2 / 3) \sum_{\mu=1}^{3}\left(P_{f \mu} \psi_{g}(0), \psi_{g_{\mu}}^{\prime}(0)\right)} \\
& =\frac{1+\sum_{n=1}^{\infty} b_{n}(\Lambda) \alpha^{2 n}}{1+\sum_{n=1}^{\infty}\left(b_{n}(\Lambda)+c_{n}(\Lambda)\right) \alpha^{2 n}} \\
& =\left(1+\sum_{n=1}^{\infty} b_{n}(\Lambda) \alpha^{2 n}\right) \\
& \cdot \sum_{n=0}^{\infty}\left(-\sum_{l=1}^{\infty}\left(b_{l}(\Lambda)+c_{l}(\Lambda)\right) \alpha^{2 l}\right)^{n} .
\end{aligned}
$$

This proves the theorem.

Theorem 10. There exists strictly positive constant $C$ such that $\lim _{\Lambda \rightarrow \infty} a_{1}(\Lambda)=C$.

Proof. From (59), we have

$$
\begin{aligned}
\frac{m_{\mathrm{eff}}}{m} & =\left\{1+b_{1}(\Lambda) \alpha^{2}+O\left(\alpha^{4}\right)\right\} \\
\cdot & {\left[1-\left\{b_{1}(\Lambda)+c_{1}(\Lambda)\right\} \alpha^{2}+O\left(\alpha^{4}\right)\right]=1-c_{1}(\Lambda) } \\
\cdot & \alpha^{2}+O\left(\alpha^{4}\right) .
\end{aligned}
$$

Therefore $a_{1}(\Lambda)=-c_{1}(\Lambda)$. Since

$$
\begin{aligned}
\sum_{n=1}^{\infty} c_{n}(\Lambda) \alpha^{2 n} & =-\frac{2}{3} \sum_{\mu=1}^{3}\left(P_{f \mu} \psi_{g}, \psi_{g_{\mu}}^{\prime}(0)\right) \\
& =-\frac{2}{3} \sum_{\mu=1}^{3}\left(P_{f \mu} \sum_{n=0}^{\infty} \frac{\alpha^{n}}{n !} \varphi_{n}, \sum_{n=0}^{\infty} \frac{\alpha^{n}}{n !} \Phi_{n}^{\mu}\right)
\end{aligned}
$$

we have

$$
\begin{aligned}
& c_{1}(\Lambda)=-\frac{2}{3} \sum_{\mu=1}^{3}\left\{\frac{1}{0 ! 2 !}\left(P_{f \mu} \varphi_{0}, \Phi_{2}^{\mu}\right)+\frac{1}{1 ! 1 !}\left(P_{f \mu} \varphi_{1}, \Phi_{1}^{\mu}\right)\right. \\
& \left.+\frac{1}{2 ! 0 !}\left(P_{f \mu} \varphi_{2}, \Phi_{0}^{\mu}\right)\right\}=-\frac{2}{3} \sum_{\mu=1}^{3}\left\{\left(P_{f \mu} \varphi_{1}, \Phi_{1}^{\mu}\right)\right. \\
& \left.+\frac{1}{2}\left(P_{f \mu} \varphi_{2}, \Phi_{0}^{\mu}\right)\right\} .
\end{aligned}
$$

Substituting $p=0$ into (27) and using (25), we have

$$
\begin{aligned}
- & \frac{1}{m}\left(P_{f \mu} \Psi, \psi_{g}\right)+\left(\left(H_{0}+\alpha H_{I}\right) \Psi, \psi_{g_{\mu}}^{\prime}(0)\right) \\
& =E(0, \alpha)\left(\Psi, \psi_{g_{\mu}}^{\prime}(0)\right) .
\end{aligned}
$$

In addition, by setting $\alpha=0$, we have $-\left(P_{f \mu} / m\right) \varphi_{0}+H_{0} \Phi_{0}^{\mu}=0$. Since $P_{f \mu} \varphi_{0}=d \Gamma\left(k_{\mu}\right) \Omega=0, H_{0} \Phi_{0}^{\mu}=0$ holds. Hence we have

$$
\Phi_{0}^{\mu}=c_{0} \Omega, \quad\left(c_{0} \text { is some constant }\right) .
$$


Differentiating both sides of (63) with respect to $\alpha$, we have

$$
\begin{aligned}
& -\frac{1}{m}\left(P_{f \mu} \Psi, s-\frac{d}{d \alpha} \psi_{g}\right)+\left(H(0) \Psi, s-\frac{d}{d \alpha} \psi_{g_{\mu}}^{\prime}(0)\right) \\
& +\left(H_{I} \Psi, \psi_{g_{\mu}}^{\prime}(0)\right) \\
& =E(0, \alpha)\left(\Psi, s-\frac{d}{d \alpha} \psi_{g_{\mu}}^{\prime}(0)\right) \\
& \quad+\frac{d}{d \alpha} E(0, \alpha)\left(\Psi, \psi_{g_{\mu}}^{\prime}(0)\right) .
\end{aligned}
$$

Substituting $\alpha=0$ into both sides, we have

$$
\left(H_{0} \Psi, \Phi_{1}^{\mu}\right)=\frac{1}{m}\left(\Psi, P_{f \mu} \varphi_{1}\right)-\left(\Psi, H_{I} \Phi_{0}^{\mu}\right) .
$$

Therefore $\Phi_{1}^{\mu} \in D\left(H_{0}\right)$ and

$$
\begin{aligned}
H_{0} \Phi_{1}^{\mu} & =\frac{1}{m} P_{f \mu} \varphi_{1}-H_{I} \Phi_{0}^{\mu} \\
& =-\frac{1}{m} P_{f \mu} \frac{1}{H_{0}} H_{I} \Omega-c_{0} H_{I} \Omega .
\end{aligned}
$$

Since $-(1 / m) P_{f \mu}\left(1 / H_{0}\right) H_{I} \Omega-c_{0} H_{I} \Omega \in \mathscr{F}_{\text {fin }}$, we have

$$
\Phi_{1}^{\mu}=-\frac{1}{m} \frac{1}{H_{0}} P_{f \mu} \frac{1}{H_{0}} H_{I} \Omega-c_{0} \frac{1}{H_{0}} H_{I} \Omega+c_{1} \Omega,
$$

where $c_{1}$ is some constant. By $a_{1}(\Lambda)=-c_{1}(\Lambda),(62),(64)$, and (68), we have

$$
\begin{aligned}
a_{1}(\Lambda)= & \frac{2}{3} \sum_{\mu=1}^{3}\left(P_{f \mu} \varphi_{1}, \Phi_{1}^{\mu}\right) \\
= & \frac{2}{3 m} \sum_{\mu=1}^{3}\left(P_{f \mu} \frac{1}{H_{0}} H_{I} \Omega, \frac{1}{H_{0}} P_{f \mu} \frac{1}{H_{0}} H_{I} \Omega\right) \\
& -\frac{2 c_{0}}{3} \sum_{\mu=1}^{3}\left(P_{f \mu} \frac{1}{H_{0}} H_{I} \Omega, \frac{1}{H_{0}} H_{I} \Omega\right) \\
& -\frac{2 c_{1}}{3} \sum_{\mu=1}^{3}\left(P_{f \mu} \frac{1}{H_{0}} H_{I} \Omega, \Omega\right) .
\end{aligned}
$$

It is also seen that

$$
\begin{aligned}
& \sum_{\mu=1}^{3}\left(P_{f \mu} \frac{1}{H_{0}} H_{I} \Omega, \frac{1}{H_{0}} H_{I} \Omega\right)=\sum_{\mu=1}^{3}\left(P_{f \mu} \frac{1}{H_{0}} H_{I} \Omega, \Omega\right) \\
& \quad=0 .
\end{aligned}
$$

Thus we have

$$
\begin{aligned}
a_{1}(\Lambda) & =\frac{2}{3 m} \sum_{\mu=1}^{3}\left(P_{f \mu} \frac{1}{H_{0}} H_{I} \Omega, \frac{1}{H_{0}} P_{f \mu} \frac{1}{H_{0}} H_{I} \Omega\right) \\
& =\frac{2}{3 m} \sum_{\mu=1}^{3}\left(P_{f \mu} \frac{1}{H_{0}} A^{+} \Omega, \frac{1}{H_{0}} P_{f_{\mu}} \frac{1}{H_{0}} A^{+} \Omega\right) \\
& =\frac{2}{3 m} \int \frac{|\widehat{\phi}(k)|^{2}}{\omega(k)} \frac{|k|^{2}}{E(k)^{3}} d k .
\end{aligned}
$$

Changing variables into polar coordinate, we have

$$
\begin{aligned}
& \frac{2}{3 m} \int \frac{|\widehat{\phi}(k)|^{2}}{\omega(k)} \frac{|k|^{2}}{E(k)^{3}} d k \\
& =\frac{8 \pi}{3 m(2 \pi)^{3}} \int_{\mathcal{K}}^{\Lambda} \frac{r^{4}}{\omega(r) F(r)^{3}} d r .
\end{aligned}
$$

Since $r^{4} / \omega(r) F(r)^{3}=O\left(r^{-3}\right)(r \rightarrow \infty)$, the improper integral $\int_{\mathcal{K}}^{\infty}\left(r^{4} / \omega(r) F(r)^{3}\right) d r$ converges. It is trivial to see that $\lim _{\Lambda \rightarrow \infty} a_{1}(\Lambda)>0$. Thus the theorem follows.

Lemma 11. It follows that $\Phi_{n}^{\mu} \in \mathscr{F}_{\text {fin }}$ for $n \in \mathbb{N} \cup\{0\}$.

Proof. By (64), we have $\Phi_{0}^{\mu} \in \mathscr{F}_{\text {fin }}$. Assume that $\Phi_{n}^{\mu} \in \mathscr{F}_{\text {fin }}$ holds when $n \leq k-1$. Differentiating both sides of (63) $k$ times with respect to $\alpha$ and substituting $\alpha=0$, we have

$$
\begin{aligned}
- & \frac{1}{m}\left(P_{f \mu} \Psi, \varphi_{k}\right)+\left(H_{0} \Psi, \Phi_{k}^{\mu}\right)+k\left(H_{I} \Psi, \Phi_{k-1}^{\mu}\right) \\
= & \sum_{j=1}^{k}\left(\begin{array}{l}
k \\
j
\end{array}\right) E_{j}\left(\Psi, \Phi_{k-j}^{\mu}\right) ;
\end{aligned}
$$

however, $E_{j}=0$ when $j$ is odd. Since $\Phi_{k-1}^{\mu} \in \mathscr{F}_{\text {fin }}, \Phi_{k-1}^{\mu} \in$ $D\left(H_{I}\right)$ and

$$
\begin{aligned}
\left(H_{0} \Psi, \Phi_{k}^{\mu}\right)= & \frac{1}{m}\left(\Psi, P_{f \mu} \varphi_{k}\right)-k\left(\Psi, H_{I} \Phi_{k-1}^{\mu}\right) \\
& +\left(\Psi, \sum_{j=1}^{k}\left(\begin{array}{l}
k \\
j
\end{array}\right) E_{j} \Phi_{k-j}^{\mu}\right) .
\end{aligned}
$$

Thus $\Phi_{k}^{\mu} \in D\left(H_{0}\right)$ and

$$
H_{0} \Phi_{k}^{\mu}=\frac{1}{m} P_{f \mu} \varphi_{k}-k H_{I} \Phi_{k-1}^{\mu}+\sum_{j=1}^{k}\left(\begin{array}{l}
k \\
j
\end{array}\right) E_{j} \Phi_{k-j}^{\mu} .
$$

Since $\varphi_{k} \in \mathscr{F}_{\text {fin }}, H_{I} \Phi_{k-1}^{\mu} \in \mathscr{F}_{\text {fin }}$, and $\Phi_{k-j}^{\mu} \in \mathscr{F}_{\text {fin }}(j=1, \ldots$, $k)$, by the assumption of induction, $H_{0} \Phi_{k}^{\mu} \in \mathscr{F}_{\text {fin }}$. Hence $\Phi_{n}^{\mu} \in$ $\mathscr{F}_{\text {fin }}$ holds when $n=k$.

Lemma 12. It holds that $\Phi_{0}^{\mu}=c_{0} \Omega, \Phi_{1}^{\mu}=-(1 / m)\left(1 / H_{0}\right) P_{f \mu}(1 /$ $\left.H_{0}\right) H_{I} \Omega-c_{0}\left(1 / H_{0}\right) H_{I} \Omega+c_{1} \Omega$, and the recurrence formulas

$$
\begin{array}{r}
\Phi_{2 l}^{\mu}=\frac{1}{H_{0}}\left\{\frac{1}{m} P_{f \mu} \varphi_{2 l}-2 l H_{I} \Phi_{2 l-1}^{\mu}\right. \\
\left.+\sum_{j=1}^{l}\left(\begin{array}{l}
2 l \\
2 j
\end{array}\right) E_{2 j} \Phi_{2 l-2 j}^{\mu}\right\}+c_{2 l} \Omega
\end{array}
$$

$\left(l \geq 1, c_{2 l}\right.$ is some constant). 


$$
\begin{gathered}
\Phi_{2 l+1}^{\mu}=\frac{1}{H_{0}}\left\{\frac{1}{m} P_{f \mu} \varphi_{2 l+1}-(2 l+1) H_{I} \Phi_{2 l}^{\mu}\right. \\
\left.+\sum_{j=1}^{l}\left(\begin{array}{c}
2 l+1 \\
2 j
\end{array}\right) E_{2 j} \Phi_{2 l+1-2 j}^{\mu}\right\}+c_{2 l+1} \Omega, \\
\left(l \geq 0, c_{2 l+1} \text { is some constant. }\right)
\end{gathered}
$$

Proof. The first and second expressions are proven in Theorem 10. From (75), it follows that

$$
\begin{aligned}
H_{0} \Phi_{2 l}^{\mu}= & \frac{1}{m} P_{f \mu} \varphi_{2 l}-2 l H_{I} \Phi_{2 l-1}^{\mu} \\
& +\sum_{j=1}^{l}\left(\begin{array}{l}
2 l \\
2 j
\end{array}\right) E_{2 j} \Phi_{2 l-2 j}^{\mu} \quad(l \geq 1) \\
H_{0} \Phi_{2 l+1}^{\mu}= & \frac{1}{m} P_{f \mu} \varphi_{2 l+1}-(2 l+1) H_{I} \Phi_{2 l}^{\mu} \\
& +\sum_{j=1}^{l}\left(\begin{array}{c}
2 l+1 \\
2 j
\end{array}\right) E_{2 j} \Phi_{2 l+1-2 j}^{\mu} \quad(l \geq 0) .
\end{aligned}
$$

These prove the lemma.

Lemma 13. It is proven that $a_{2}(\Lambda)$ can be expanded as

$$
\begin{aligned}
a_{2}(\Lambda)= & \frac{2}{3 m} \sum_{j=1}^{8} I_{j}(\Lambda)+\frac{E_{2}(\Lambda)}{m} I_{9}(\Lambda)-a_{1}(\Lambda) I_{10}(\Lambda) \\
& +a_{1}(\Lambda)^{2},
\end{aligned}
$$

where $I_{j}$ are given by

$$
\begin{aligned}
& I_{1}(\Lambda)=\frac{1}{4} \iint \frac{\left|\widehat{\phi}_{1}\right|^{2}\left|\widehat{\phi}_{2}\right|^{2}}{\omega_{1} \omega_{2}}\left(\frac{\left|k_{1}\right|^{2}}{E_{1}^{3}}+\frac{\left|k_{2}\right|^{2}}{E_{2}^{3}}\right) \\
& \cdot\left(\frac{1}{E_{1}}+\frac{1}{E_{2}}\right) \frac{1}{E_{12}} d k_{1} d k_{2}, \\
& I_{2}(\Lambda)=\frac{1}{8} \iint \frac{\left|\widehat{\phi}_{1}\right|^{2}\left|\widehat{\phi}_{2}\right|^{2}}{\omega_{1} \omega_{2}}\left(\frac{\left|k_{1}\right|^{2}}{E_{1}^{4}}+\frac{\left|k_{2}\right|^{2}}{E_{2}^{4}}\right) \frac{1}{E_{12}} d k_{1} d k_{2}, \\
& I_{3}(\Lambda)=\frac{1}{8} \iint \frac{\left|\widehat{\phi}_{1}\right|^{2}\left|\widehat{\phi}_{2}\right|^{2}}{\omega_{1} \omega_{2}}\left(\frac{1}{E_{1}^{2}}+\frac{1}{E_{2}^{2}}\right)\left(\frac{1}{E_{1}}+\frac{1}{E_{2}}\right) \\
& \cdot \frac{\left(k_{1}, k_{2}\right)}{E_{12}^{2}} d k_{1} d k_{2}, \\
& I_{4}(\Lambda)=\frac{1}{4} \iint \frac{\left|\widehat{\phi}_{1}\right|^{2}\left|\widehat{\phi}_{2}\right|^{2}}{\omega_{1} \omega_{2}}\left(\frac{\left|k_{1}\right|^{2}}{E_{1}^{2}}+\frac{\left|k_{2}\right|^{2}}{E_{2}^{2}}\right) \\
& \cdot\left(\frac{1}{E_{1}}+\frac{1}{E_{2}}\right) \frac{1}{E_{12}^{2}} d k_{1} d k_{2},
\end{aligned}
$$

$$
\begin{gathered}
I_{5}(\Lambda)=\frac{1}{4} \iint \frac{\left|\widehat{\phi}_{1}\right|^{2}\left|\widehat{\phi}_{2}\right|^{2}}{\omega_{1} \omega_{2} E_{1}^{2} E_{2}^{2}} \frac{\left(k_{1}, k_{2}\right)}{E_{12}} d k_{1} d k_{2}, \\
I_{6}(\Lambda)=\frac{1}{8} \iint \frac{\left|\widehat{\phi}_{1}\right|^{2}\left|\widehat{\phi}_{2}\right|^{2}}{\omega_{1} \omega_{2}}\left(\frac{1}{E_{1}}+\frac{1}{E_{2}}\right)^{2} \\
\cdot \frac{\left|k_{1}\right|^{2}+\left|k_{2}\right|^{2}}{E_{12}^{3}} d k_{1} d k_{2},
\end{gathered}
$$

$$
I_{7}(\Lambda)=\frac{1}{4} \iint \frac{\left|\widehat{\phi}_{1}\right|^{2}\left|\widehat{\phi}_{2}\right|^{2}}{\omega_{1} \omega_{2}}\left(\frac{1}{E_{1}}+\frac{1}{E_{2}}\right)^{2}
$$$$
\frac{\left(k_{1}, k_{2}\right)}{E_{12}^{3}} d k_{1} d k_{2}
$$$$
I_{8}(\Lambda)=\frac{1}{4} \iint \frac{\left|\widehat{\phi}_{1}\right|^{2}\left|\widehat{\phi}_{2}\right|^{2}}{\omega_{1} \omega_{2}}\left(\frac{1}{E_{1}}+\frac{1}{E_{2}}\right) \frac{\left(k_{1}, k_{2}\right)}{E_{12}^{4}} d k_{1} d k_{2}
$$$$
I_{9}(\Lambda)=\frac{1}{2} \int \frac{|\widehat{\phi}(k)|^{2}|k|^{2}}{\omega(k) E(k)^{4}} d k
$$$$
I_{10}(\Lambda)=\frac{1}{2} \int \frac{|\widehat{\phi}(k)|^{2}}{\omega(k) E(k)^{2}} d k \text {. }
$$

The proof of Lemma 13 is given in the next section. The asymptotic behaviors of terms $I_{j}(\Lambda)$ as $\Lambda \rightarrow \infty$ is given in the lemma below. Only two terms $I_{1}(\Lambda)$ and $I_{2}(\Lambda)$ logarithmically diverge, and other terms converge as $\Lambda \rightarrow \infty$.

Lemma 14. (1)-(3) follow the following:

(1) There exist some constants $C_{3}$ and $C_{4}$ such that $C_{3} \leq$ $\lim _{\Lambda \rightarrow \infty}\left(I_{1}(\Lambda) / \log \Lambda\right) \leq C_{4}$.

(2) There exist some constants $C_{5}$ and $C_{6}$ such that $C_{5} \leq$ $\lim _{\Lambda \rightarrow \infty}\left(I_{2}(\Lambda) / \log \Lambda\right) \leq C_{6}$.

(3) For $j=3,4,5,6,7,8 \lim _{\Lambda \rightarrow \infty}\left|I_{j}(\Lambda)\right|<\infty$.

The proof of Lemma 14 is technical and also given in the next section.

Lemma 15. It holds that

$$
\lim _{\Lambda \rightarrow \infty} \frac{E_{2}(\Lambda)}{\log \Lambda}=-\frac{m}{\pi^{2}} .
$$

Proof. From (36), we have $E_{2}(\Lambda)=-\left(1 / 2 \pi^{2}\right) \int_{\kappa}^{\Lambda}\left(r^{2} / \omega(r) F(r)\right) d r$ and $\lim _{r \rightarrow \infty}\left(\left(r^{2} / \omega(r) F(r)\right) /(1 / r)\right)=2 m$. It implies $(80)$.

Now we are in the position to state the main theorem in this paper.

Theorem 16. There exist some constants $C_{1}$ and $C_{2}$ such that

$$
C_{1} \leq \lim _{\Lambda \rightarrow \infty} \frac{a_{2}(\Lambda)}{\log \Lambda} \leq C_{2}
$$


Proof. We have $I_{9}(\Lambda)=\left(1 / 4 \pi^{2}\right) \int_{\mathcal{K}}^{\Lambda}\left(r^{4} / \omega(r) F(r)^{4}\right) d r$. Since $r^{4} / \omega(r) F(r)^{4}=O\left(r^{-5}\right)(r \rightarrow \infty)$, we have

$$
\lim _{\Lambda \rightarrow \infty}\left|I_{9}(\Lambda)\right|<\infty
$$

We also have $I_{10}(\Lambda)=\left(1 / 4 \pi^{2}\right) \int_{\mathcal{K}}^{\Lambda}\left(r^{2} / \omega(r) F(r)^{2}\right) d r$. Then $r^{2} / \omega(r) F(r)^{2}=O\left(r^{-3}\right)(r \rightarrow \infty)$ and we also have

$$
\lim _{\Lambda \rightarrow \infty}\left|I_{10}(\Lambda)\right|<\infty \text {. }
$$

By (82) and (83), Theorem 10, and Lemmas 13, 14, and 15 we can conclude the theorem.

\section{Proof of Lemmas 13 and 14}

In this section we prove Lemmas 13 and 14.

4.1. Proof of Lemma 13. From (59) and $a_{1}(\Lambda)=-c_{1}(\Lambda)$, we have $a_{2}(\Lambda)=-c_{2}(\Lambda)-b_{1}(\Lambda) a_{1}(\Lambda)+a_{1}(\Lambda)^{2}$. Here

$$
\begin{aligned}
& b_{1}(\Lambda)=\left(\varphi_{1}, \varphi_{1}\right)=\frac{1}{2} \int \frac{|\widehat{\phi}(k)|^{2}}{\omega(k) E(k)^{2}} d k \\
& c_{2}(\Lambda)=-\frac{2}{3}\left\{\frac{1}{0 ! 4 !} \sum_{\mu=1}^{3}\left(P_{f \mu} \varphi_{0}, \Phi_{4}^{\mu}\right)\right. \\
& +\frac{1}{1 ! 3 !} \sum_{\mu=1}^{3}\left(P_{f \mu} \varphi_{1}, \Phi_{3}^{\mu}\right)+\frac{1}{2 ! 2 !} \sum_{\mu=1}^{3}\left(P_{f \mu} \varphi_{2}, \Phi_{2}^{\mu}\right) \\
& \left.+\frac{1}{3 ! 1 !} \sum_{\mu=1}^{3}\left(P_{f \mu} \varphi_{3}, \Phi_{1}^{\mu}\right)+\frac{1}{4 ! 0 !} \sum_{\mu=1}^{3}\left(P_{f_{\mu}} \varphi_{4}, \Phi_{0}^{\mu}\right)\right\} \\
& \quad=-\frac{1}{9} \sum_{\mu=1}^{3}\left(P_{f \mu} \varphi_{1}, \Phi_{3}^{\mu}\right)-\frac{1}{6} \sum_{\mu=1}^{3}\left(P_{f \mu} \varphi_{2}, \Phi_{2}^{\mu}\right)-\frac{1}{9} \\
& \quad \sum_{\mu=1}^{3}\left(P_{f_{\mu}} \varphi_{3}, \Phi_{1}^{\mu}\right) .
\end{aligned}
$$

Using recurrence formulas (33), (34), and (76), we have

$$
\begin{aligned}
\varphi_{2}= & 2\left(\frac{1}{H_{0}} H_{I}\right)^{2} \Omega, \\
\varphi_{3}= & -6\left(\frac{1}{H_{0}} H_{I}\right)^{3} \Omega-3 E_{2}\left(\frac{1}{H_{0}}\right)^{2} H_{I} \Omega, \\
\Phi_{2}^{\mu}= & \frac{2}{m} \frac{1}{H_{0}} P_{f \mu}\left(\frac{1}{H_{0}} H_{I}\right)^{2} \Omega \\
& +\frac{2}{m} \frac{1}{H_{0}} H_{I} \frac{1}{H_{0}} P_{f \mu} \frac{1}{H_{0}} H_{I} \Omega \\
& +2 c_{0}\left(\frac{1}{H_{0}} H_{I}\right)^{2} \Omega-2 c_{1} \frac{1}{H_{0}} H_{I} \Omega+c_{2} \Omega,
\end{aligned}
$$

$$
\begin{aligned}
\Phi_{3}^{\mu}= & -\frac{6}{m} \frac{1}{H_{0}} P_{f \mu}\left(\frac{1}{H_{0}} H_{I}\right)^{3} \Omega \\
& -\frac{6}{m} \frac{1}{H_{0}} H_{I} \frac{1}{H_{0}} P_{f \mu}\left(\frac{1}{H_{0}} H_{I}\right)^{2} \Omega \\
& -\frac{6}{m}\left(\frac{1}{H_{0}} H_{I}\right)^{2} \frac{1}{H_{0}} P_{f \mu} \frac{1}{H_{0}} H_{I} \Omega \\
& -\frac{3}{m} E_{2} \frac{1}{H_{0}} P_{f \mu}\left(\frac{1}{H_{0}}\right)^{2} H_{I} \Omega \\
& -\frac{3}{m} E_{2}\left(\frac{1}{H_{0}}\right)^{2} P_{f_{\mu}} \frac{1}{H_{0}} H_{I} \Omega \\
& -6 c_{0}\left(\frac{1}{H_{0}} H_{I}\right)^{3} \Omega+6 c_{1}\left(\frac{1}{H_{0}} H_{I}\right)^{2} \Omega \\
& -3 c_{2} \frac{1}{H_{0}} H_{I} \Omega-3 c_{0} E_{2}\left(\frac{1}{H_{0}}\right)^{2} H_{I} \Omega+c_{3} \Omega .
\end{aligned}
$$

Substituting them into (85), we have

$$
\begin{aligned}
c_{2}(\Lambda) & -\frac{2}{3 m}\left\{\sum_{\mu=1}^{3}\left(P_{f \mu} \frac{1}{H_{0}} H_{I} \Omega, \frac{1}{H_{0}} P_{f_{\mu}}\left(\frac{1}{H_{0}} H_{I}\right)^{3} \Omega\right)\right. \\
& +\sum_{\mu=1}^{3}\left(P_{f \mu} \frac{1}{H_{0}} H_{I} \Omega, \frac{1}{H_{0}} H_{I} \frac{1}{H_{0}} P_{f \mu}\left(\frac{1}{H_{0}} H_{I}\right)^{2} \Omega\right) \\
& +\sum_{\mu=1}^{3}\left(P_{f \mu} \frac{1}{H_{0}} H_{I} \Omega,\left(\frac{1}{H_{0}} H_{I}\right)^{2} \frac{1}{H_{0}} P_{f_{\mu}} \frac{1}{H_{0}} H_{I} \Omega\right) \\
& +\sum_{\mu=1}^{3}\left(P_{f \mu}\left(\frac{1}{H_{0}} H_{I}\right)^{2} \Omega, \frac{1}{H_{0}} P_{f_{\mu}}\left(\frac{1}{H_{0}} H_{I}\right)^{2} \Omega\right) \\
& +\sum_{\mu=1}^{3}\left(P_{f_{\mu}}\left(\frac{1}{H_{0}} H_{I}\right)^{2} \Omega, \frac{1}{H_{0}} H_{I} \frac{1}{H_{0}} P_{f_{\mu}} \frac{1}{H_{0}} H_{I} \Omega\right) \\
& +\sum_{\mu=1}^{3}\left(P_{f \mu} \frac{1}{H_{0}} H_{I} \Omega,\left(\frac{1}{H_{0}} H_{I}\right)^{3} \Omega\right)+\frac{2 c_{1}}{3}\left(P_{f \mu}\left(\frac{1}{H_{0}}\right)^{2} H_{I} \Omega, \frac{1}{H_{0}} P_{f \mu} \frac{1}{H_{0}} H_{I} \Omega\right)^{3}-\frac{2 c_{0}}{3} \\
+ & \left.\sum_{\mu=1}^{3}\left(P_{f_{\mu}}\left(\frac{1}{H_{0}} H_{I}\right)^{3} \Omega, \frac{1}{H_{0}} P_{f \mu} \frac{1}{H_{0}} H_{I} \Omega\right)\right\} \\
+ & \frac{E_{2}}{3 m}\left\{\sum_{\mu=1}^{3}\left(P_{f \mu} \frac{1}{H_{0}} H_{I} \Omega,\left(\frac{1}{H_{0}}\right)^{2} P_{f \mu} \frac{1}{H_{0}} H_{I} \Omega\right)^{2} \frac{1}{H_{0}} P_{f \mu}\left(\frac{1}{H_{0}}\right)^{2} H_{I} \Omega\right)
\end{aligned}
$$




$$
\begin{aligned}
& \cdot \sum_{\mu=1}^{3}\left(P_{f \mu} \frac{1}{H_{0}} H_{I} \Omega,\left(\frac{1}{H_{0}} H_{I}\right)^{2} \Omega\right)-\frac{c_{2}}{3} \\
& \cdot \sum_{\mu=1}^{3}\left(P_{f \mu} \frac{1}{H_{0}} H_{I} \Omega, \frac{1}{H_{0}} H_{I} \Omega\right)-\frac{c_{0} E_{2}}{3} \\
& \cdot \sum_{\mu=1}^{3}\left(P_{f \mu} \frac{1}{H_{0}} H_{I} \Omega,\left(\frac{1}{H_{0}}\right)^{2} H_{I} \Omega\right)+\frac{c_{3}}{9} \\
& \cdot \sum_{\mu=1}^{3}\left(P_{f \mu} \frac{1}{H_{0}} H_{I} \Omega, \Omega\right)-\frac{2 c_{0}}{3} \\
& \cdot \sum_{\mu=1}^{3}\left(P_{f \mu}\left(\frac{1}{H_{0}} H_{I}\right)^{2} \Omega,\left(\frac{1}{H_{0}} H_{I}\right)^{2} \Omega\right)+\frac{2 c_{1}}{3} \\
& \cdot \sum_{\mu=1}^{3}\left(P_{f \mu}\left(\frac{1}{H_{0}} H_{I}\right)^{2} \Omega, \frac{1}{H_{0}} H_{I} \Omega\right)-\frac{c_{2}}{3} \\
& \cdot \sum_{\mu=1}^{3}\left(P_{f \mu}\left(\frac{1}{H_{0}} H_{I}\right)^{2} \Omega, \Omega\right)-\frac{2 c_{0}}{3} \\
& \cdot \sum_{\mu=1}^{3}\left(P_{f \mu}\left(\frac{1}{H_{0}} H_{I}\right)^{3} \Omega, \frac{1}{H_{0}} H_{I} \Omega\right)+\frac{2 c_{1}}{3} \\
& \cdot \sum_{\mu=1}^{3}\left(P_{f \mu}\left(\frac{1}{H_{0}} H_{I}\right)^{3} \Omega, \Omega\right)-\frac{c_{0} E_{2}}{3} \\
& \cdot \sum_{\mu=1}^{3}\left(P_{f \mu}\left(\frac{1}{H_{0}}\right)^{2} H_{I} \Omega, \frac{1}{H_{0}} H_{I} \Omega\right)+\frac{c_{1} E_{2}}{3} \\
& \cdot \sum_{\mu=1}^{3}\left(P_{f \mu}\left(\frac{1}{H_{0}}\right)^{2} H_{I} \Omega, \Omega\right)=\sum_{j=1}^{21}(j) .
\end{aligned}
$$

$$
\begin{aligned}
& =\sum_{\mu=1}^{3}\left(P_{f \mu}\left(\frac{1}{H_{0}} H_{I}\right)^{2} \Omega,\left(\frac{1}{H_{0}} H_{I}\right)^{2} \Omega\right) \\
= & \sum_{\mu=1}^{3}\left(P_{f \mu}\left(\frac{1}{H_{0}} H_{I}\right)^{2} \Omega, \frac{1}{H_{0}} H_{I} \Omega\right) \\
= & \sum_{\mu=1}^{3}\left(P_{f \mu}\left(\frac{1}{H_{0}} H_{I}\right)^{2} \Omega, \Omega\right) \\
= & \sum_{\mu=1}^{3}\left(P_{f \mu}\left(\frac{1}{H_{0}} H_{I}\right)^{3} \Omega, \frac{1}{H_{0}} H_{I} \Omega\right) \\
= & \sum_{\mu=1}^{3}\left(P_{f \mu}\left(\frac{1}{H_{0}} H_{I}\right)^{3} \Omega, \Omega\right) \\
= & \sum_{\mu=1}^{3}\left(P_{f \mu}\left(\frac{1}{H_{0}}\right)^{2} H_{I} \Omega, \frac{1}{H_{0}} H_{I} \Omega\right) \\
= & \sum_{\mu=1}^{3}\left(P_{f \mu}\left(\frac{1}{H_{0}}\right)^{2} H_{I} \Omega, \Omega\right)=0 .
\end{aligned}
$$

We can compute remaining terms (1)-(9) as

$$
\text { (1) } \begin{aligned}
& \sum_{\mu=1}^{3}\left(P_{f \mu} \frac{1}{H_{0}} H_{I} \Omega, \frac{1}{H_{0}} P_{f \mu}\left(\frac{1}{H_{0}} H_{I}\right)^{3} \Omega\right) \\
= & \sum_{\mu=1}^{3}\left(A^{+} \frac{1}{H_{0}} P_{f \mu} \frac{1}{H_{0}} P_{f \mu} \frac{1}{H_{0}} A^{+} \Omega,\left(\frac{1}{H_{0}} A^{+}\right)^{2} \Omega\right) \\
= & \frac{1}{8} \iint \frac{\left|\hat{\phi}_{1}\right|^{2}\left|\hat{\phi}_{2}\right|^{2}}{\omega_{1} \omega_{2}}\left(\frac{\left|k_{1}\right|^{2}}{E_{1}^{3}}+\frac{\left|k_{2}\right|^{2}}{E_{2}^{3}}\right)\left(\frac{1}{E_{1}}+\frac{1}{E_{2}}\right) \\
\cdot & \frac{1}{E_{12}} d k_{1} d k_{2}, \\
\text { (2) } & \sum_{\mu=1}^{3}\left(P_{f \mu} \frac{1}{H_{0}} H_{I} \Omega, \frac{1}{H_{0}} H_{I} \frac{1}{H_{0}} P_{f \mu}\left(\frac{1}{H_{0}} H_{I}\right)^{2} \Omega\right) \\
= & \sum_{\mu=1}^{3}\left(A^{+} \frac{1}{H_{0}} P_{f \mu} \frac{1}{H_{0}} A^{+} \Omega, \frac{1}{H_{0}} P_{f \mu}\left(\frac{1}{H_{0}} A^{+}\right)^{2} \Omega\right) \\
= & \frac{1}{8} \iint \frac{\left|\hat{\phi}_{1}\right|^{2}\left|\widehat{\phi}_{2}\right|^{2}}{\omega_{1} \omega_{2}}\left(\frac{\left|k_{1}\right|^{2}}{E_{1}^{2}}+\frac{\left|k_{2}\right|^{2}}{E_{2}^{2}}\right)\left(\frac{1}{E_{1}}+\frac{1}{E_{2}}\right) \\
& \left.\frac{1}{E_{12}^{2}} d k_{1} d k_{2}+\frac{1}{8} \iint \frac{\left|k_{1}, k_{2}\right|^{2}\left|\widehat{\phi}_{2}\right|^{2}}{\omega_{1} \omega_{2}} d \frac{1}{E_{1}^{2}}+\frac{1}{E_{2}^{2}}\right)^{2} d k_{1}, \\
& \left(k_{2},\right.
\end{aligned}
$$




$$
\begin{array}{rlrl} 
& \sum_{\mu=1}^{3}\left(P_{f \mu} \frac{1}{H_{0}} H_{I} \Omega,\left(\frac{1}{H_{0}} H_{I}\right)^{2} \frac{1}{H_{0}} P_{f \mu} \frac{1}{H_{0}} H_{I} \Omega\right) & \text { (7) } \sum_{\mu=1}^{3}\left(P_{f \mu} \frac{1}{H_{0}} H_{I} \Omega, \frac{1}{H_{0}} P_{f \mu}\left(\frac{1}{H_{0}}\right)^{2} H_{I} \Omega\right) \\
= & \sum_{\mu=1}^{3}\left(A^{+} \frac{1}{H_{0}} P_{f \mu} \frac{1}{H_{0}} A^{+} \Omega, \frac{1}{H_{0}} A^{+} \frac{1}{H_{0}} P_{f \mu} \frac{1}{H_{0}} A^{+} \Omega\right) & =\frac{1}{2} \int \frac{|\widehat{\phi}(k)|^{2}|k|^{2}}{\omega(k) E(k)^{4}} d k, \\
= & \frac{1}{8} \iint \frac{\left|\widehat{\phi}_{1}\right|^{2}\left|\widehat{\phi}_{2}\right|^{2}}{\omega_{1} \omega_{2}}\left(\frac{\left|k_{1}\right|^{2}}{E_{1}^{4}}+\frac{\left|k_{2}\right|^{2}}{E_{2}^{4}}\right) \frac{1}{E_{12}} d k_{1} d k_{2} & \text { (8) } \sum_{\mu=1}^{3}\left(P_{f \mu} \frac{1}{H_{0}} H_{I} \Omega,\left(\frac{1}{H_{0}}\right)^{2} P_{f \mu} \frac{1}{H_{0}} H_{I} \Omega\right) \\
+ & \frac{1}{4} \iint \frac{\left|\widehat{\phi}_{1}\right|^{2}\left|\widehat{\phi}_{2}\right|^{2}}{\omega_{1} \omega_{2} E_{1}^{2} E_{2}^{2}} \frac{\left(k_{1}, k_{2}\right)}{E_{12}} d k_{1} d k_{2}, & =\frac{1}{2} \int \frac{|\widehat{\phi}(k)|^{2}|k|^{2}}{\omega(k) E(k)^{4}} d k, \\
\text { (4) } \sum_{\mu=1}^{3}\left(P_{f \mu}\left(\frac{1}{H_{0}} H_{I}\right)^{2} \Omega, \frac{1}{H_{0}} P_{f \mu}\left(\frac{1}{H_{0}} H_{I}\right)^{2} \Omega\right) & \text { (9) } \sum_{\mu=1}^{3}\left(P_{f \mu}\left(\frac{1}{H_{0}}\right)^{2} H_{I} \Omega, \frac{1}{H_{0}} P_{f \mu} \frac{1}{H_{0}} H_{I} \Omega\right) \\
= & \sum_{\mu=1}^{3}\left(P_{f \mu}\left(\frac{1}{H_{0}} A^{+}\right)^{2} \Omega, \frac{1}{H_{0}} P_{f \mu}\left(\frac{1}{H_{0}} A^{+}\right)^{2} \Omega\right) & =\frac{1}{2} \int \frac{|\widehat{\phi}(k)|^{2}|k|^{2}}{\omega(k) E(k)^{4}} d k . \\
= & \frac{1}{8} \iint \frac{\left|\hat{\phi}_{1}\right|^{2}\left|\hat{\phi}_{2}\right|^{2}}{\omega_{1} \omega_{2}}\left(\frac{1}{E_{1}}+\frac{1}{E_{2}}\right)^{2} & \text { Thus the lemma follows. }
\end{array}
$$$$
\frac{\left|k_{1}\right|^{2}+\left|k_{2}\right|^{2}}{E_{12}^{3}} d k_{1} d k_{2}+\frac{1}{4}
$$$$
\iint \frac{\left|\widehat{\phi}_{1}\right|^{2}\left|\widehat{\phi}_{2}\right|^{2}}{\omega_{1} \omega_{2}}\left(\frac{1}{E_{1}}+\frac{1}{E_{2}}\right)^{2} \frac{\left(k_{1}, k_{2}\right)}{E_{12}^{3}} d k_{1} d k_{2}
$$$$
\text { (5) } \sum_{\mu=1}^{3}\left(P_{f \mu}\left(\frac{1}{H_{0}} H_{I}\right)^{2} \Omega, \frac{1}{H_{0}} H_{I} \frac{1}{H_{0}} P_{f \mu} \frac{1}{H_{0}} H_{I} \Omega\right)
$$$$
=\sum_{\mu=1}^{3}\left(P_{f \mu}\left(\frac{1}{H_{0}} A^{+}\right)^{2} \Omega, \frac{1}{H_{0}} A^{+} \frac{1}{H_{0}} P_{f \mu} \frac{1}{H_{0}} A^{+} \Omega\right)
$$$$
=\frac{1}{8} \iint \frac{\left|\widehat{\phi}_{1}\right|^{2}\left|\widehat{\phi}_{2}\right|^{2}}{\omega_{1} \omega_{2}}\left(\frac{\left|k_{1}\right|^{2}}{E_{1}^{2}}+\frac{\left|k_{2}\right|^{2}}{E_{2}^{2}}\right)\left(\frac{1}{E_{1}}+\frac{1}{E_{2}}\right)
$$$$
\cdot \frac{1}{E_{12}^{2}} d k_{1} d k_{2}+\frac{1}{4} \iint \frac{\left|\widehat{\phi}_{1}\right|^{2}\left|\widehat{\phi}_{2}\right|^{2}}{\omega_{1} \omega_{2}}\left(\frac{1}{E_{1}}+\frac{1}{E_{2}}\right)
$$$$
\cdot \frac{\left(k_{1}, k_{2}\right)}{E_{12}^{4}} d k_{1} d k_{2}
$$$$
\text { (6) } \sum_{\mu=1}^{3}\left(P_{f \mu}\left(\frac{1}{H_{0}} H_{I}\right)^{3} \Omega, \frac{1}{H_{0}} P_{f \mu} \frac{1}{H_{0}} H_{I} \Omega\right)
$$$$
=\sum_{\mu=1}^{3}\left(\left(\frac{1}{H_{0}} A^{+}\right)^{2} \Omega, A^{+} \frac{1}{H_{0}} P_{f \mu} \frac{1}{H_{0}} P_{f \mu} \frac{1}{H_{0}} A^{+} \Omega\right)
$$$$
=\frac{1}{8} \iint \frac{\left|\hat{\phi}_{1}\right|^{2}\left|\hat{\phi}_{2}\right|^{2}}{\omega_{1} \omega_{2}}\left(\frac{\left|k_{1}\right|^{2}}{E_{1}^{3}}+\frac{\left|k_{2}\right|^{2}}{E_{2}^{3}}\right)\left(\frac{1}{E_{1}}+\frac{1}{E_{2}}\right)
$$$$
\cdot \frac{1}{E_{12}} d k_{1} d k_{2}
$$

Thus the lemma follows.

\subsection{Proof of Lemma 14}

Proof of $C_{3} \leq \lim _{\Lambda \rightarrow \infty}\left(I_{1}(\Lambda) / \log \Lambda\right)$ and $C_{5} \leq \lim _{\Lambda \rightarrow \infty}\left(I_{2}(\Lambda) /\right.$ $\log \Lambda$ ). Changing variables to polar coordinates, we have

$$
\begin{aligned}
& I_{1}(\Lambda)=\frac{2 \pi^{2}}{(2 \pi)^{6}} \\
& \cdot \int_{-1}^{1} \int_{\mathcal{K}}^{\Lambda} \int_{\kappa}^{\Lambda} \frac{r_{1}^{2} r_{2}^{2}}{\omega\left(r_{1}\right) \omega\left(r_{2}\right)}\left(\frac{r_{1}^{2}}{F\left(r_{1}\right)^{3}}+\frac{r_{2}^{2}}{F\left(r_{2}\right)^{3}}\right) \\
& \cdot\left(\frac{1}{F\left(r_{1}\right)}+\frac{1}{F\left(r_{2}\right)}\right) \frac{1}{L\left(r_{1}, r_{2}, z\right)} d z d r_{1} d r_{2},
\end{aligned}
$$

where

$$
L\left(r_{1}, r_{2}, z\right)=\frac{r_{1}^{2}+r_{2}^{2}+2 r_{1} r_{2} z}{2 m}+\omega\left(r_{1}\right)+\omega\left(r_{2}\right) .
$$

We define $h\left(r_{1}, r_{2}\right), h_{1}\left(r_{1}, r_{2}\right), h_{2}\left(r_{1}, r_{2}\right), S(\Lambda), S_{1}(\Lambda)$ and $S_{2}(\Lambda)$ as

$$
\begin{aligned}
& h\left(r_{1}, r_{2}\right)=\frac{r_{1}^{2} r_{2}^{2}}{\omega\left(r_{1}\right) \omega\left(r_{2}\right)}\left(\frac{r_{1}^{2}}{F\left(r_{1}\right)^{3}}+\frac{r_{2}^{2}}{F\left(r_{2}\right)^{3}}\right) \\
& \cdot\left(\frac{1}{F\left(r_{1}\right)}+\frac{1}{F\left(r_{2}\right)}\right) \frac{1}{L\left(r_{1}, r_{2}, 1\right)}, \\
& h_{1}\left(r_{1}, r_{2}\right)=\frac{r_{1}^{2} r_{2}^{4}}{\omega\left(r_{1}\right) \omega\left(r_{2}\right) F\left(r_{2}\right)^{4} L\left(r_{1}, r_{2}, 1\right)}, \\
& h_{2}\left(r_{1}, r_{2}\right)=h\left(r_{1}, r_{2}\right)-h_{1}\left(r_{1}, r_{2}\right),
\end{aligned}
$$




$$
\begin{aligned}
& S(\Lambda)=\int_{\mathcal{K}}^{\Lambda} \int_{\mathcal{K}}^{\Lambda} h\left(r_{1}, r_{2}\right) d r_{1} d r_{2} \\
& S_{1}(\Lambda)=\int_{\mathcal{K}}^{\kappa+1} \int_{\mathcal{K}+1+\nu+m}^{\Lambda} h_{1}\left(r_{1}, r_{2}\right) d r_{2} d r_{1} \\
& S_{2}(\Lambda)=\int_{\mathcal{K}}^{\mathcal{\kappa}+1} \int_{\mathcal{K}}^{\mathcal{\kappa}+1+\nu+m} h_{1}\left(r_{1}, r_{2}\right) d r_{2} d r_{1} \\
& \quad+\int_{\mathcal{K}+1}^{\Lambda} \int_{\mathcal{K}}^{\Lambda} h_{1}\left(r_{1}, r_{2}\right) d r_{1} d r_{2} \\
& \quad+\int_{\mathcal{K}}^{\Lambda} \int_{\mathcal{K}}^{\Lambda} h_{2}\left(r_{1}, r_{2}\right) d r_{1} d r_{2} .
\end{aligned}
$$

Then

$$
\frac{4 \pi^{2}}{(2 \pi)^{6}} S(\Lambda) \leq I_{1}(\Lambda) .
$$

In addition, $S(\Lambda)=S_{1}(\Lambda)+S_{2}(\Lambda)$ follows. Since $h_{1}\left(r_{1}, r_{2}\right)>0$ and $h_{2}\left(r_{1}, r_{2}\right)>0, S_{2}(\Lambda)>0$. Hence

$$
S(\Lambda)>S_{1}(\Lambda)
$$

Let $r_{2}$ satisfy $\kappa \leq r_{2} \leq \kappa+1$. Suppose that $\kappa+1+\nu+m \leq$ $r_{1} \leq \Lambda$. Since $v<r_{1}, r_{1}^{2}+v^{2}<2 r_{1}^{2}$ holds. Therefore we have $\omega\left(r_{1}\right)<\sqrt{2} r_{1}$. Since $r_{2}<r_{1}$, we have $r_{1} r_{2}<r_{1}^{2}$ and $r_{2}^{2}<r_{1}^{2}$. Thus $L\left(r_{1}, r_{2}, 1\right)<2(1 / m+\sqrt{2}) r_{1}^{2}$. So,

$$
\begin{aligned}
& \int_{\kappa+1+\nu+m}^{\Lambda} \frac{r_{1}^{2}}{\omega\left(r_{1}\right) L\left(r_{1}, r_{2}, 1\right)} d r_{1} \\
& \quad>\frac{1}{2 \sqrt{2}(1 / m+\sqrt{2})} \int_{\kappa+1+\nu+m}^{\Lambda} r_{1}^{-1} d r_{1} \\
& \quad=\frac{1}{2 \sqrt{2}(1 / m+2)}(\log \Lambda-\log (\kappa+1+\nu+m))
\end{aligned}
$$

follows. When $\kappa \leq r_{2} \leq \kappa+1$, we have

$$
\begin{aligned}
& \omega\left(r_{2}\right) \leq \sqrt{(\kappa+1)^{2}+v^{2}}, \\
& F\left(r_{2}\right) \leq \frac{(\kappa+1)^{2}}{2 m}+\sqrt{(\kappa+1)^{2}+v^{2}} .
\end{aligned}
$$

Then

$$
\begin{aligned}
& S_{1}(\Lambda)=\int_{\mathcal{K}}^{\kappa+1} \frac{r_{2}^{4}}{\omega\left(r_{2}\right) F\left(r_{2}\right)^{4}} d r_{2} \\
& \cdot \int_{\mathcal{\kappa}+1+\nu+m}^{\Lambda} \frac{r_{1}^{2}}{\omega\left(r_{1}\right) L\left(r_{1}, r_{2}, 1\right)} d r_{1} \\
& >\frac{K}{2 \sqrt{2}(1 / m+\sqrt{2})}(\log \Lambda-\log (\kappa+1+\nu+m)) \\
& \cdot \int_{\mathcal{K}}^{\kappa+1} r_{2}^{4} d r_{2}=\frac{K\left\{(\kappa+1)^{5}-\kappa^{5}\right\}}{10 \sqrt{2}(1 / m+\sqrt{2})}(\log \Lambda \\
& -\log (\kappa+1+\nu+m))
\end{aligned}
$$

where

$$
\begin{aligned}
& K \\
& =\frac{1}{\sqrt{(\kappa+1)^{2}+v^{2}}\left((\kappa+1)^{2} / 2 m+\sqrt{(\kappa+1)^{2}+v^{2}}\right)^{4}} .
\end{aligned}
$$

From (93), (94), and (97), $C_{3} \leq \lim _{\Lambda \rightarrow \infty}\left(I_{1}(\Lambda) / \log \Lambda\right)$ follows.

The proof of $C_{5} \leq \lim _{\Lambda \rightarrow \infty}\left(I_{2}(\Lambda) / \log \Lambda\right)$ is similar to that of $C_{3} \leq \lim _{\Lambda \rightarrow \infty}\left(I_{1}(\Lambda) / \log \Lambda\right)$. Then we omit it.

Proof of $\lim _{\Lambda \rightarrow \infty}\left(I_{1}(\Lambda) / \log \Lambda\right) \leq C_{4}$. We redefine $h\left(r_{1}, r_{2}\right)$, $h_{1}\left(r_{1}, r_{2}\right), h_{2}\left(r_{1}, r_{2}\right), S_{1}(\Lambda), S_{2}(\Lambda)$, and $S(\Lambda)$ as

$$
\begin{aligned}
& h\left(r_{1}, r_{2}\right)=\frac{r_{1}^{2} r_{2}^{2}}{\omega\left(r_{1}\right) \omega\left(r_{2}\right)}\left(\frac{r_{1}^{2}}{F\left(r_{1}\right)^{3}}+\frac{r_{2}^{2}}{F\left(r_{2}\right)^{3}}\right) \\
& \cdot\left(\frac{1}{F\left(r_{1}\right)}+\frac{1}{F\left(r_{2}\right)}\right) \frac{1}{L\left(r_{1}, r_{2},-1\right)}, \\
& h_{1}\left(r_{1}, r_{2}\right)=\frac{r_{1}^{2} r_{2}^{4}}{\omega\left(r_{1}\right) \omega\left(r_{2}\right) F\left(r_{2}\right)^{4} L\left(r_{1}, r_{2},-1\right)}, \\
& h_{2}\left(r_{1}, r_{2}\right)=\frac{r_{1}^{2} r_{2}^{4}}{\omega\left(r_{1}\right) \omega\left(r_{2}\right) F\left(r_{1}\right) F\left(r_{2}\right)^{3} L\left(r_{1}, r_{2},-1\right)} \\
& S_{1}(\Lambda)=\int_{\mathcal{K}}^{\Lambda} \int_{\mathcal{K}}^{\Lambda} h_{1}\left(r_{1}, r_{2}\right) d r_{1} d r_{2}, \\
& S_{2}(\Lambda)=\int_{\mathcal{K}}^{\Lambda} \int_{\mathcal{K}}^{\Lambda} h_{2}\left(r_{1}, r_{2}\right) d r_{1} d r_{2}, \\
& S(\Lambda)=\int_{\mathcal{K}}^{\Lambda} \int_{\mathcal{K}}^{\Lambda} h\left(r_{1}, r_{2}\right) d r_{1} d r_{2} .
\end{aligned}
$$

Then we have

$$
I_{1}(\Lambda) \leq \frac{4 \pi^{2}}{(2 \pi)^{6}} S(\Lambda) .
$$

Since $h\left(r_{1}, r_{2}\right)=h_{1}\left(r_{1}, r_{2}\right)+h_{1}\left(r_{2}, r_{1}\right)+h_{2}\left(r_{1}, r_{2}\right)+h_{2}\left(r_{2}, r_{1}\right)$, we have

$$
S(\Lambda)=2\left(S_{1}(\Lambda)+S_{2}(\Lambda)\right)
$$

Let $B$ be $B=\left((\kappa+1)^{3}-\kappa^{3}\right) / 6 v^{2}$. Since $v<\omega\left(r_{1}\right)$ and $2 v<$ $L\left(r_{1}, r_{2},-1\right)$,

$$
\int_{\kappa}^{\kappa+1} \frac{r_{1}^{2}}{\omega\left(r_{1}\right) L\left(r_{1}, r_{2},-1\right)} d r_{1}<B
$$

follows. Let $Y$ be $Y=2 r_{2}+\kappa+1$. Since $r_{1}<\omega\left(r_{1}\right)$ and $r_{1}<$ $L\left(r_{1}, r_{2},-1\right)$,

$$
\int_{\kappa+1}^{Y} \frac{r_{1}^{2}}{\omega\left(r_{1}\right) L\left(r_{1}, r_{2},-1\right)} d r_{1}<2 r_{2}
$$


holds. When $Y \leq r_{1}$, since $2 r_{2}<r_{1}$, we have $r_{1}-r_{2}>r_{1} / 2$. Then $L\left(r_{1}, r_{2},-1\right)>\left(r_{1}-r_{2}\right)^{2} / 2 m>r_{1}^{2} / 8 m$. So,

$$
\int_{Y}^{\Lambda} \frac{r_{1}^{2}}{\omega\left(r_{1}\right) L\left(r_{1}, r_{2},-1\right)} d r_{1}<8 m \int_{Y}^{\Lambda} \frac{d r_{1}}{r_{1}}<8 m \log \Lambda
$$

follows. From (102), (103), and (104), we have

$$
\int_{\mathcal{K}}^{\Lambda} \frac{r_{1}^{2}}{\omega\left(r_{1}\right) L\left(r_{1}, r_{2},-1\right)} d r_{1}<B+2 r_{2}+8 m \log \Lambda
$$

Using this, we see that $S_{1}(\Lambda)<\int_{\mathcal{K}}^{\Lambda}\left(B+2 r_{2}+8 m \log \Lambda\right)\left(r_{2}^{4} /\right.$ $\left.\omega\left(r_{2}\right) F\left(r_{2}\right)^{4}\right) d r_{2}$. Since $r_{2}<\omega\left(r_{2}\right)$ and $r_{2}^{2} / 2 m<F\left(r_{2}\right)$, we have

$$
\begin{aligned}
S_{1}(\Lambda)< & \int_{\kappa}^{\Lambda}\left(2 r_{2}+B+8 m \log \Lambda\right) \frac{16 m^{4}}{r_{2}^{5}} d r_{2} \\
= & \frac{32 m^{4}}{3}\left(\frac{1}{\kappa^{3}}-\frac{1}{\Lambda^{3}}\right) \\
& +4 m^{4}(B+8 m \log \Lambda)\left(\frac{1}{\kappa^{4}}-\frac{1}{\Lambda^{4}}\right) \\
< & \frac{32 m^{5}}{\kappa^{4}} \log \Lambda+\frac{32 m^{4}}{3 \kappa^{3}}+\frac{4 m^{4} B}{\kappa^{4}} .
\end{aligned}
$$

Since $r_{1}<L\left(r_{1}, r_{2},-1\right)$,

$$
\begin{aligned}
\int_{\mathcal{\kappa}}^{\Lambda} & \frac{r_{1}^{2}}{\omega\left(r_{1}\right) F\left(r_{1}\right) L\left(r_{1}, r_{2},-1\right)} d r_{1}<2 m \int_{\kappa}^{\Lambda} \frac{d r_{1}}{r_{1}^{2}} \\
& <\frac{2 m}{\kappa} .
\end{aligned}
$$

Then we have

$$
\begin{aligned}
& S_{2}(\Lambda)=\int_{\mathcal{\kappa}}^{\Lambda} \frac{r_{2}^{4}}{\omega\left(r_{2}\right) F\left(r_{2}\right)^{3}} d r_{2} \\
& \cdot \int_{\mathcal{K}}^{\Lambda} \frac{r_{1}^{2}}{\omega\left(r_{1}\right) F\left(r_{1}\right) L\left(r_{1}, r_{2},-1\right)} d r_{1}<\frac{2 m}{\kappa} \\
& \cdot \int_{\kappa}^{\Lambda} \frac{r_{2}^{4}}{\omega\left(r_{2}\right) F\left(r_{2}\right)^{3}} d r_{2}<\frac{16 m^{4}}{\kappa} \int_{\kappa}^{\Lambda} \frac{d r_{2}}{r_{2}^{3}}<\frac{8 m^{4}}{\kappa^{3}} .
\end{aligned}
$$

From (101), (106), and (108), it follows that

$$
S(\Lambda)<\frac{64 m^{5}}{\kappa^{4}} \log \Lambda+\frac{112 m^{4}}{3 \kappa^{3}}+\frac{8 m^{2} B}{\kappa^{4}} .
$$

From (100) and (109), the lemma follows.
Proof of $\lim _{\Lambda \rightarrow \infty}\left(I_{2}(\Lambda) / \log \Lambda\right) \leq C_{6}$. We redefine $h\left(r_{1}, r_{2}\right)$, $h_{1}\left(r_{1}, r_{2}\right), S(\Lambda)$, and $S_{1}(\Lambda)$ as

$$
\begin{aligned}
& h\left(r_{1}, r_{2}\right) \\
& =\frac{r_{1}^{2} r_{2}^{2}}{\omega\left(r_{1}\right) \omega\left(r_{2}\right)}\left(\frac{r_{1}^{2}}{F\left(r_{1}\right)^{4}}+\frac{r_{2}^{2}}{F\left(r_{2}\right)^{4}}\right) \frac{1}{L\left(r_{1}, r_{2},-1\right)}, \\
& h_{1}\left(r_{1}, r_{2}\right)=\frac{r_{1}^{2} r_{2}^{4}}{\omega\left(r_{1}\right) \omega\left(r_{2}\right) F\left(r_{2}\right)^{4} L\left(r_{1}, r_{2},-1\right)}, \\
& S(\Lambda)=\int_{\mathcal{K}}^{\Lambda} \int_{\mathcal{K}}^{\Lambda} h\left(r_{1}, r_{2}\right) d r_{1} d r_{2}, \\
& S_{1}(\Lambda)=\int_{\mathcal{K}}^{\Lambda} \int_{\mathcal{K}}^{\Lambda} h_{1}\left(r_{1}, r_{2}\right) d r_{1} d r_{2} .
\end{aligned}
$$

We have

$$
\begin{aligned}
& I_{2}(\Lambda)=\frac{\pi^{2}}{(2 \pi)^{6}} \\
& \cdot \int_{-1}^{1} \int_{\mathcal{K}}^{\Lambda} \int_{\mathcal{K}}^{\Lambda} \frac{r_{1}^{2} r_{2}^{2}}{\omega\left(r_{1}\right) \omega\left(r_{2}\right)}\left(\frac{r_{1}^{2}}{F\left(r_{1}\right)^{4}}+\frac{r_{2}^{2}}{F\left(r_{2}\right)^{4}}\right) \\
& \cdot \frac{1}{L\left(r_{1}, r_{2}, z\right)} d z d r_{1} d r_{2} .
\end{aligned}
$$

Then

$$
I_{2}(\Lambda) \leq \frac{2 \pi^{2}}{(2 \pi)^{6}} S(\Lambda)
$$

holds. Since $h\left(r_{1}, r_{2}\right)=h_{1}\left(r_{1}, r_{2}\right)+h_{1}\left(r_{2}, r_{1}\right)$, we have

$$
S(\Lambda)=2 S_{1}(\Lambda) .
$$

We have

$$
\int_{\kappa}^{\Lambda} \frac{r_{1}^{2}}{\omega\left(r_{1}\right) L\left(r_{1}, r_{2},-1\right)} d r_{1}<B+2 r_{2}+8 m \log \Lambda
$$

in the same way as the proof of $\lim _{\Lambda \rightarrow \infty}\left(I_{1}(\Lambda) / \log \Lambda\right) \leq C_{4}$. Since $r_{2}<\omega\left(r_{2}\right)$ and $r_{2}^{2} / 2 m<F\left(r_{2}\right)$, we have

$$
\begin{aligned}
S_{1}(\Lambda)< & \int_{\kappa}^{\Lambda}\left(B+2 r_{2}+8 m \log \Lambda\right) \frac{r_{2}^{4}}{\omega\left(r_{2}\right) F\left(r_{2}\right)^{4}} d r_{2} \\
< & 32 m^{4} \int_{\mathcal{\kappa}}^{\Lambda} \frac{d r_{2}}{r_{2}^{4}} \\
& +16 m^{4}(B+8 m \log \Lambda) \int_{\kappa}^{\Lambda} \frac{d r_{2}}{r_{2}^{5}} \\
< & \frac{32 m^{5}}{\kappa^{4}} \log \Lambda+\frac{32 m^{4}}{3 \kappa^{3}}+\frac{4 m^{4} B}{\kappa^{4}} .
\end{aligned}
$$

From (112), (113), and (115), the lemma follows. 
Proof of $\lim _{\Lambda \rightarrow \infty}\left(I_{3}(\Lambda) / \log \Lambda\right)=0$. We define $h\left(r_{1}, r_{2}, z\right)$ as

$$
\begin{aligned}
h\left(r_{1}, r_{2}, z\right)= & \frac{z r_{1}^{3} r_{2}^{3}}{\omega\left(r_{1}\right) \omega\left(r_{2}\right)}\left(\frac{1}{F\left(r_{1}\right)^{2}}+\frac{1}{F\left(r_{2}\right)^{2}}\right) \\
& \cdot\left(\frac{1}{F\left(r_{1}\right)}+\frac{1}{F\left(r_{2}\right)}\right) \frac{1}{L\left(r_{1}, r_{2}, z\right)^{2}}
\end{aligned}
$$

and redefine $S(\Lambda)$ as

$$
S(\Lambda)=\int_{-1}^{1} \int_{\mathcal{K}}^{\Lambda} \int_{\mathcal{K}}^{\Lambda} h\left(r_{1}, r_{2}, z\right) d z d r_{2} d r_{1}
$$

Then we have $I_{3}(\Lambda)=\left(\pi^{2} /(2 \pi)^{6}\right) S(\Lambda)$. We divide $S(\Lambda)$ in the following way.

$S(\Lambda)$

$$
\begin{aligned}
= & \int_{-1}^{0} \int_{\mathcal{K}}^{\Lambda} \int_{\mathcal{K}}^{\Lambda} h\left(r_{1}, r_{2}, z\right) d z d r_{2} d r_{1} \\
& +\int_{0}^{1} \int_{\mathcal{K}}^{\Lambda} \int_{\mathcal{K}}^{\Lambda} h\left(r_{1}, r_{2}, z\right) d z d r_{2} d r_{1} \\
= & -\int_{1}^{0} \int_{\mathcal{K}}^{\Lambda} \int_{\mathcal{K}}^{\Lambda} h\left(r_{1}, r_{2},-z\right) d z d r_{2} d r_{1} \\
& +\int_{0}^{1} \int_{\mathcal{K}}^{\Lambda} \int_{\mathcal{K}}^{\Lambda} h\left(r_{1}, r_{2}, z\right) d z d r_{2} d r_{1} \\
= & \int_{0}^{1} \int_{\mathcal{K}}^{\Lambda} \int_{\mathcal{K}}^{\Lambda}\left(h\left(r_{1}, r_{2}, z\right)+h\left(r_{1}, r_{2},-z\right)\right) d z d r_{2} d r_{1} \\
= & \int_{0}^{1} \int_{\mathcal{K}}^{\Lambda} \int_{\mathcal{K}}^{\Lambda} g\left(r_{1}, r_{2}, z\right) d z d r_{2} d r_{1},
\end{aligned}
$$

where

$$
\begin{aligned}
g( & \left.r_{1}, r_{2}, z\right)=h\left(r_{1}, r_{2}, z\right)+h\left(r_{1}, r_{2},-z\right) \\
= & -\frac{2 z^{2} r_{1}^{4} r_{2}^{4}}{m \omega\left(r_{1}\right) \omega\left(r_{2}\right)}\left(\frac{1}{F\left(r_{1}\right)^{2}}+\frac{1}{F\left(r_{2}\right)^{2}}\right) \\
& \cdot\left(\frac{1}{F\left(r_{1}\right)}+\frac{1}{F\left(r_{2}\right)}\right) \\
& \cdot \frac{\left(\left(r_{1}^{2}+r_{2}^{2}\right) / m+2 \omega\left(r_{1}\right)+2 \omega\left(r_{2}\right)\right)}{L\left(r_{1}, r_{2}, z\right)^{2} L\left(r_{1}, r_{2},-z\right)^{2}} .
\end{aligned}
$$

Since $g\left(r_{1}, r_{2}, z\right) \leq 0, S(\Lambda)$ is decreasing in $\Lambda$.

$$
\begin{aligned}
S(\Lambda)= & \int_{0}^{1} \int_{\mathcal{K}}^{\Lambda} \int_{r_{2}}^{\Lambda} g\left(r_{1}, r_{2}, z\right) d z d r_{2} d r_{1} \\
& +\int_{0}^{1} \int_{\mathcal{K}}^{\Lambda} \int_{r_{1}}^{\Lambda} g\left(r_{1}, r_{2}, z\right) d z d r_{1} d r_{2} \\
= & 2 \int_{0}^{1} \int_{\mathcal{K}}^{\Lambda} \int_{r_{2}}^{\Lambda} g\left(r_{1}, r_{2}, z\right) d z d r_{2} d r_{1} \\
= & 2 \int_{0}^{1} \int_{\mathcal{K}}^{\Lambda} \int_{r_{2}}^{2 r_{2}} g\left(r_{1}, r_{2}, z\right) d z d r_{2} d r_{1} \\
& +2 \int_{0}^{1} \int_{\mathcal{K}}^{\Lambda} \int_{2 r_{2}}^{\Lambda} g\left(r_{1}, r_{2}, z\right) d z d r_{2} d r_{1} .
\end{aligned}
$$

Since $\kappa \leq r_{1}$, we have $1 \leq r_{1} / \kappa$. Hence $r_{1}^{2}+v^{2} \leq\left(\left(\kappa^{2}+\right.\right.$ $\left.\left.v^{2}\right) / \kappa^{2}\right) r_{1}^{2}$. Therefore we have $\omega\left(r_{1}\right) \leq\left(\sqrt{\kappa^{2}+v^{2}} / \kappa\right) r_{1}$, and similarly $\omega\left(r_{2}\right) \leq\left(\sqrt{\kappa^{2}+v^{2}} / \kappa\right) r_{2}$. When $0 \leq z \leq 1$, we have

$$
L\left(r_{1}, r_{2}, z\right)>\frac{r_{1}^{2}}{2 m}
$$

Then

$$
\begin{aligned}
L\left(r_{1}, r_{2},-z\right)= & \frac{\left(r_{1}-r_{2}\right)^{2}+2 r_{1} r_{2}(1-z)}{2 m}+\omega\left(r_{1}\right) \\
& +\omega\left(r_{2}\right)>\omega\left(r_{1}\right)>r_{1} .
\end{aligned}
$$

When $r_{2} \leq r_{1}$, we have $r_{2}^{2} / m \leq r_{1}^{2} / m$. Then it holds that

$$
\begin{aligned}
& 2 \omega\left(r_{1}\right) \leq \frac{2 r_{1}^{2}}{\kappa^{2}} \sqrt{\kappa^{2}+\nu^{2}}, \\
& 2 \omega\left(r_{2}\right) \leq \frac{2 r_{2}}{\kappa} \sqrt{\kappa^{2}+v^{2}} \leq \frac{2 r_{1}^{2}}{\kappa^{2}} \sqrt{\kappa^{2}+v^{2}} .
\end{aligned}
$$

Thus we have

$$
\begin{aligned}
& \frac{r_{1}^{2}}{m}+\frac{r_{2}^{2}}{m}+2 \omega\left(r_{1}\right)+2 \omega\left(r_{2}\right) \\
& \quad \leq 2\left(\frac{1}{m}+\frac{2 \sqrt{\kappa^{2}+v^{2}}}{\kappa^{2}}\right) r_{1}^{2} .
\end{aligned}
$$

When $r_{2} \leq r_{1} \leq 2 r_{2}$, we have

$$
\begin{gathered}
\frac{1}{F\left(r_{1}\right)}+\frac{1}{F\left(r_{2}\right)}<\frac{10 m}{r_{1}^{2}}, \\
\frac{1}{F\left(r_{1}\right)^{2}}+\frac{1}{F\left(r_{2}\right)^{2}}<\frac{68 m^{2}}{r_{1}^{4}} .
\end{gathered}
$$


From (119), (121), (122), (124), and (125), it follows that

$$
\begin{aligned}
& -g\left(r_{1}, r_{2}, z\right) \leq \frac{2}{m} \frac{z^{2} r_{1}^{4} r_{2}^{4}}{r_{1} r_{2}} \frac{68 m^{2}}{r_{1}^{4}} \frac{10 m}{r_{1}^{2}} \\
& \cdot 2\left(\frac{1}{m}+\frac{2 \sqrt{\kappa^{2}+v^{2}}}{\kappa^{2}}\right) r_{1}^{2}\left(\frac{2 m}{r_{1}^{2}}\right)^{2} \frac{1}{r_{1}^{2}} \\
& =68 \cdot 10 \cdot 16 m^{4}\left(\frac{1}{m}+\frac{2 \sqrt{\kappa^{2}+v^{2}}}{\kappa^{2}}\right) \frac{z^{2} r_{2}^{3}}{r_{1}^{7}} .
\end{aligned}
$$

Hence

$$
\begin{gathered}
-2 \int_{0}^{1} \int_{\kappa}^{\Lambda} \int_{r_{2}}^{2 r_{2}} g\left(r_{1}, r_{2}, z\right) d z d r_{2} d r_{1} \\
\leq 1190 \frac{m^{4}}{\kappa^{2}}\left(\frac{1}{m}+\frac{2 \sqrt{\kappa^{2}+\nu^{2}}}{\kappa^{2}}\right) .
\end{gathered}
$$

When $2 r_{2} \leq r_{1}$, we have

$$
\begin{gathered}
\frac{1}{F\left(r_{1}\right)}+\frac{1}{F\left(r_{2}\right)}<\frac{5 m}{2 r_{2}^{2}}, \\
\frac{1}{F\left(r_{1}\right)^{2}}+\frac{1}{F\left(r_{2}\right)^{2}}<\frac{17 m^{2}}{4 r_{2}^{4}} .
\end{gathered}
$$

Since $r_{2} \leq r_{1} / 2$, we have $r_{1} / 2 \leq r_{1}-r_{2}$. Then we have

$$
\begin{gathered}
L\left(r_{1}, r_{2}, \pm z\right)=\frac{\left(r_{1}-r_{2}\right)^{2}+2 r_{1} r_{2}(1 \pm z)}{2 m}+\omega\left(r_{1}\right) \\
+\omega\left(r_{2}\right)>\frac{\left(r_{1}-r_{2}\right)^{2}}{2 m} \geq \frac{r_{1}^{2}}{8 m} .
\end{gathered}
$$

From (119), (124), (128), and (129), it follows that

$$
\begin{aligned}
& -g\left(r_{1}, r_{2}, z\right) \leq \frac{2}{m} \frac{z^{2} r_{1}^{4} r_{2}^{4}}{r_{1} r_{2}} \frac{17 m^{2}}{4 r_{2}^{4}} \frac{5 m}{2 r_{2}^{2}} \\
& \cdot 2\left(\frac{1}{m}+\frac{2 \sqrt{\kappa^{2}+v^{2}}}{\kappa^{2}}\right) r_{1}^{2}\left(\frac{8 m}{r_{1}^{2}}\right)^{4}=2^{11} \cdot 5 \\
& \cdot 17 m^{6}\left(\frac{1}{m}+\frac{2 \sqrt{\kappa^{2}+v^{2}}}{\kappa^{2}}\right) \frac{z^{2}}{r_{1}^{3} r_{2}^{3}} .
\end{aligned}
$$

Then by (120), (127), and (131), we have

$$
\begin{aligned}
-S(\Lambda) \leq & \frac{1190 m^{4}}{\kappa^{2}}\left(\frac{1}{m}+\frac{2 \sqrt{\kappa^{2}+v^{2}}}{\kappa^{2}}\right) \\
& +2^{7} \cdot 5 \cdot 17 \frac{m^{6}}{\kappa^{4}}\left(\frac{1}{m}+\frac{2 \sqrt{\kappa^{2}+v^{2}}}{\kappa^{2}}\right) .
\end{aligned}
$$

Since $S(\Lambda)$ is decreasing and bounded below, it converges as $\Lambda \rightarrow \infty$. This fact proves the lemma.
Proof of $\lim _{\Lambda \rightarrow \infty}\left(I_{4}(\Lambda) / \log \Lambda\right)=0$. We redefine $h\left(r_{1}, r_{2}, z\right)$ as

$$
\begin{aligned}
h\left(r_{1}, r_{2}, z\right)= & \frac{r_{1}^{2} r_{2}^{2}}{\omega\left(r_{1}\right) \omega\left(r_{2}\right)}\left(\frac{r_{1}^{2}}{F\left(r_{1}\right)^{2}}+\frac{r_{2}^{2}}{F\left(r_{2}\right)^{2}}\right) \\
& \cdot\left(\frac{1}{F\left(r_{1}\right)}+\frac{1}{F\left(r_{2}\right)}\right) \frac{1}{L\left(r_{1}, r_{2}, z\right)^{2}} .
\end{aligned}
$$

Then we have $I_{4}(\Lambda)=\left(2 \pi^{2} /(2 \pi)^{6}\right) \int_{-1}^{1} \int_{\mathcal{K}}^{\Lambda} \int_{\mathcal{K}}^{\Lambda} h\left(r_{1}, r_{2}, z\right) d z d r_{1} d r_{2}$. We define $J(\Lambda)$ as

$$
J(\Lambda)=\int_{-1}^{1} \int_{\mathcal{K}}^{\Lambda} \int_{\mathcal{K}}^{\Lambda} h\left(r_{1}, r_{2}, z\right) d z d r_{1} d r_{2} .
$$

Step 1. We define $S(\Lambda, z)$ as

$$
S(\Lambda, z)=\int_{\mathcal{K}}^{\Lambda} \int_{\mathcal{K}}^{\Lambda} h\left(r_{1}, r_{2}, z\right) d r_{1} d r_{2} .
$$

Our first task is to prove that $\lim _{\Lambda \rightarrow \infty} S(\Lambda, z)$ exists for all $z \epsilon$ $I=[-1,1]$. Since $h\left(r_{1}, r_{2}, z\right)>0, S(\Lambda, z)$ is increasing in $\Lambda$. Let

$$
\begin{aligned}
& S_{1}(\Lambda, z)=2 \int_{\mathcal{K}}^{\Lambda} \int_{r_{2}}^{2 r_{2}} h\left(r_{1}, r_{2}, z\right) d r_{2} d r_{1}, \\
& S_{2}(\Lambda, z)=2 \int_{\mathcal{K}}^{\Lambda} \int_{2 r_{2}}^{\Lambda} h\left(r_{1}, r_{2}, z\right) d r_{2} d r_{1} .
\end{aligned}
$$

Then

$$
\begin{aligned}
S(\Lambda, z)= & \int_{\mathcal{K}}^{\Lambda} \int_{r_{2}}^{\Lambda} h\left(r_{1}, r_{2}, z\right) d r_{2} d r_{1} \\
& +\int_{\mathcal{K}}^{\Lambda} \int_{r_{1}}^{\Lambda} h\left(r_{1}, r_{2}, z\right) d r_{1} d r_{2} \\
= & 2 \int_{\mathcal{K}}^{\Lambda} \int_{r_{2}}^{\Lambda} h\left(r_{1}, r_{2}, z\right) d r_{2} d r_{1} \\
= & 2 \int_{\mathcal{K}}^{\Lambda} \int_{r_{2}}^{2 r_{2}} h\left(r_{1}, r_{2}, z\right) d r_{2} d r_{1} \\
& +2 \int_{\mathcal{K}}^{\Lambda} \int_{2 r_{2}}^{\Lambda} h\left(r_{1}, r_{2}, z\right) d r_{2} d r_{1} \\
= & S_{1}(\Lambda, z)+S_{2}(\Lambda, z)
\end{aligned}
$$

holds. We have

$$
\begin{aligned}
\frac{1}{F\left(r_{1}\right)}+\frac{1}{F\left(r_{2}\right)} & <2 m\left(\frac{1}{r_{1}^{2}}+\frac{1}{r_{2}^{2}}\right), \\
\frac{r_{1}^{2}}{F\left(r_{1}\right)^{2}}+\frac{r_{2}^{2}}{F\left(r_{2}\right)^{2}} & <4 m^{2}\left(\frac{1}{r_{1}^{2}}+\frac{1}{r_{2}^{2}}\right), \\
r_{1} & <L\left(r_{1}, r_{2}, z\right) .
\end{aligned}
$$


Let $r_{2} \leq r_{1} \leq 2 r_{2}$. Since $1 / r_{2}^{2} \leq 4 / r_{1}^{2}$, it holds that

$$
\frac{1}{r_{1}^{2}}+\frac{1}{r_{2}^{2}} \leq \frac{5}{r_{1}^{2}}
$$

Then from (138) and (140), it follows that

$$
\begin{gathered}
\frac{1}{F\left(r_{1}\right)}+\frac{1}{F\left(r_{2}\right)}<\frac{10 m}{r_{1}^{2}}, \\
\frac{r_{1}^{2}}{F\left(r_{1}\right)^{2}}+\frac{r_{2}^{2}}{F\left(r_{2}\right)^{2}}<\frac{20 m^{2}}{r_{1}^{2}} .
\end{gathered}
$$

Hence from (139) and (141), it follows that $h\left(r_{1}, r_{2}, z\right)<$ $200 m^{3} r_{2} / r_{1}^{5}$. Therefore we have

$$
\begin{aligned}
S_{1}(\Lambda, z) & <400 m^{3} \int_{\kappa}^{\Lambda} r_{2} d r_{2} \int_{r_{2}}^{2 r_{2}} \frac{d r_{1}}{r_{1}^{5}} \\
& =\frac{375 m^{3}}{8}\left(\frac{1}{\kappa^{2}}-\frac{1}{\Lambda^{2}}\right)<\frac{375 m^{3}}{8 \kappa^{2}} .
\end{aligned}
$$

Let $2 r_{2} \leq r_{1}$. Since $r_{1} / 2 \leq r_{1}-r_{2}$, we have

$$
\frac{r_{1}^{2}}{8 m} \leq \frac{\left(r_{1}-r_{2}\right)^{2}}{2 m}<L\left(r_{1}, r_{2}, z\right) \text {. }
$$

Since $r_{2}^{2} \leq r_{1}^{2}$, we have

$$
\begin{gathered}
\frac{1}{F\left(r_{1}\right)}+\frac{1}{F\left(r_{2}\right)}<\frac{4 m}{r_{2}^{2}}, \\
\frac{r_{1}^{2}}{F\left(r_{1}\right)^{2}}+\frac{r_{2}^{2}}{F\left(r_{2}\right)^{2}}<\frac{8 m^{2}}{r_{2}^{2}} .
\end{gathered}
$$

Hence from (143) and (144), it follows that $h\left(r_{1}, r_{2}, z\right)<$ $2048 m^{5} / r_{1}^{3} r_{2}^{3}$. Therefore we have

$$
\begin{aligned}
S_{2}(\Lambda, z) & <4096 m^{5} \int_{\kappa}^{\Lambda} \frac{d r_{2}}{r_{2}^{3}} \int_{2 r_{2}}^{\Lambda} \frac{d r_{1}}{r_{1}^{3}} \\
& <512 m^{5} \int_{\kappa}^{\Lambda} \frac{d r_{2}}{r_{2}^{5}}<\frac{128 m^{5}}{\kappa^{4}} .
\end{aligned}
$$

From (137), (142), and (145), it follows that

$$
S(\Lambda, z)<\frac{375 m^{3}}{8 \kappa^{2}}+\frac{128 m^{5}}{\kappa^{4}} .
$$

Since $S(\Lambda, z)$ is increasing in $\Lambda$ and bounded above for all $z \in$ $I$, it converges as $\Lambda$ goes to infinity.

Step 2. Our second task is to prove that $J(\Lambda)$ converges when $\Lambda$ goes to infinity. Let $M\left(r_{1}, r_{2}\right)$ be

$$
\begin{aligned}
M\left(r_{1}, r_{2}\right)= & \frac{r_{1}^{2} r_{2}^{2}}{\omega\left(r_{1}\right) \omega\left(r_{2}\right)}\left(\frac{r_{1}^{2}}{F\left(r_{1}\right)^{2}}+\frac{r_{2}^{2}}{F\left(r_{2}\right)^{2}}\right) \\
& \cdot\left(\frac{1}{F\left(r_{1}\right)}+\frac{1}{F\left(r_{2}\right)}\right) \frac{1}{L\left(r_{1}, r_{2},-1\right)^{2}} .
\end{aligned}
$$

$\left|h\left(r_{1}, r_{2}, z\right)\right| \leq M\left(r_{1}, r_{2}\right)$ holds for all $\left(r_{1}, r_{2}, z\right) \in[\kappa, \Lambda]^{2} \times I$, and by Step 1 there exists

$$
\lim _{\Lambda \rightarrow \infty} \int_{\mathcal{K}}^{\Lambda} \int_{\kappa}^{\Lambda} M\left(r_{1}, r_{2}\right) d r_{1} d r_{2}
$$

Since

$$
\begin{aligned}
M_{\Lambda} & =\int_{\mathcal{K}}^{\Lambda} \int_{\mathcal{K}}^{\Lambda} M\left(r_{1}, r_{2}\right) d r_{1} d r_{2} \longrightarrow \\
M_{\infty} & =\lim _{\Lambda \rightarrow \infty} \int_{\mathcal{K}}^{\Lambda} \int_{\mathcal{K}}^{\Lambda} M\left(r_{1}, r_{2}\right) d r_{1} d r_{2},
\end{aligned}
$$

from Cauchy convergence condition, for any $\epsilon>0$, there exists $\Lambda_{0} \in[\kappa, \infty)$ such that if $\Lambda_{0}<\Lambda_{1} \leq \Lambda_{2},\left|M_{\Lambda_{2}}-M_{\Lambda_{1}}\right|<$ $\epsilon$. Then for $\Lambda_{0}<\Lambda_{1} \leq \Lambda_{2}$ and all $z \in I$,

$$
\begin{aligned}
\mid S & \left(\Lambda_{2}, z\right)-S\left(\Lambda_{1}, z\right) \mid \\
& =\mid \int_{\mathcal{K}}^{\Lambda_{1}} \int_{\Lambda_{1}}^{\Lambda_{2}} h\left(r_{1}, r_{2}, z\right) d r_{1} d r_{2} \\
& +\int_{\mathcal{K}}^{\Lambda_{1}} \int_{\Lambda_{1}}^{\Lambda_{2}} h\left(r_{1}, r_{2}, z\right) d r_{2} d r_{1} \\
& +\int_{\Lambda_{1}}^{\Lambda_{2}} \int_{\Lambda_{1}}^{\Lambda_{2}} h\left(r_{1}, r_{2}, z\right) d r_{1} d r_{2} \mid \\
& \leq \int_{\mathcal{K}}^{\Lambda_{1}} \int_{\Lambda_{1}}^{\Lambda_{2}} M\left(r_{1}, r_{2}\right) d r_{1} d r_{2} \\
& +\int_{\mathcal{K}}^{\Lambda_{1}} \int_{\Lambda_{1}}^{\Lambda_{2}} M\left(r_{1}, r_{2}\right) d r_{2} d r_{1} \\
& +\int_{\Lambda_{1}}^{\Lambda_{2}} \int_{\Lambda_{1}}^{\Lambda_{2}} M\left(r_{1}, r_{2}\right) d r_{1} d r_{2}=\left|M_{\Lambda_{2}}-M_{\Lambda_{1}}\right|<\epsilon .
\end{aligned}
$$

Therefore $\sup _{z \in I}\left|S\left(\Lambda_{2}, z\right)-S\left(\Lambda_{1}, z\right)\right| \leq\left|M_{\Lambda_{2}}-M_{\Lambda_{1}}\right|<\epsilon$ holds. Since family of functions $(S(\Lambda, \cdot))_{\Lambda \in[\kappa, \infty)}$ on $I$ satisfies uniform Cauchy conditions, it converges uniformly on $I$. Since $[\kappa, \Lambda]^{2}$ is a Jordan measurable bounded closed set of $\mathbb{R}^{2}$, the function $S(\Lambda, z)$ is continuous on $I$. Hence

$$
S(\infty, z)=\lim _{\Lambda \rightarrow \infty} \int_{\mathcal{K}}^{\Lambda} \int_{\mathcal{K}}^{\Lambda} h\left(r_{1}, r_{2}, z\right) d r_{1} d r_{2}
$$

is continuous on $I$. Since both $S(\Lambda, z)$ and $S(\infty, z)$ are integrable on Jordan measurable set $I$, by uniform convergence theorem, we have

$$
\lim _{\Lambda \rightarrow \infty} \int_{-1}^{1} S(\Lambda, z) d z=\int_{-1}^{1} S(\infty, z) d z
$$

It implies that $J(\Lambda)$ converges as $\Lambda \rightarrow \infty$. 
Proof of $\lim _{\Lambda \rightarrow \infty}\left(I_{5}(\Lambda) / \log \Lambda\right)=0$. We redefine $h\left(r_{1}, r_{2}, z\right)$, $g\left(r_{1}, r_{2}, z\right)$, and $S(\Lambda)$ as

$$
\begin{aligned}
& h\left(r_{1}, r_{2}, z\right) \\
& =\frac{z r_{1}^{3} r_{2}^{3}}{\omega\left(r_{1}\right) \omega\left(r_{2}\right) F\left(r_{1}\right)^{2} F\left(r_{2}\right)^{2} L\left(r_{1}, r_{2}, z\right)}, \\
& g\left(r_{1}, r_{2}, z\right)=h\left(r_{1}, r_{2}, z\right)+h\left(r_{1}, r_{2},-z\right), \\
& S(\Lambda)=\int_{-1}^{1} \int_{\mathcal{K}}^{\Lambda} \int_{\mathcal{K}}^{\Lambda} h\left(r_{1}, r_{2}, z\right) d z d r_{1} d r_{2} .
\end{aligned}
$$

Then $I_{5}(\Lambda)=\left(2 \pi^{2} /(2 \pi)^{6}\right) S(\Lambda)$. We have

$$
S(\Lambda)=\int_{0}^{1} \int_{\mathcal{K}}^{\Lambda} \int_{\mathcal{K}}^{\Lambda} g\left(r_{1}, r_{2}, z\right) d z d r_{2} d r_{1}
$$

in the same way as (118). Since

$$
\begin{aligned}
& g\left(r_{1}, r_{2}, z\right) \\
& =-\frac{2 z^{2} r_{1}^{4} r_{2}^{4}}{m^{2} \omega\left(r_{1}\right) \omega\left(r_{2}\right) F\left(r_{1}\right)^{2} F\left(r_{2}\right)^{2} L\left(r_{1}, r_{2}, z\right) L\left(r_{1}, r_{2},-z\right)} \\
& \leq 0,
\end{aligned}
$$

$S(\Lambda)$ is decreasing in $\Lambda$. Since $r_{1}<L\left(r_{1}, r_{2}, z\right)$, we have

$$
\frac{r_{1}^{4}}{\omega\left(r_{1}\right) F\left(r_{1}\right)^{2} L\left(r_{1}, r_{2}, z\right)}<\frac{4 m^{2}}{r_{1}^{2}} .
$$

Similarly, we have

$$
\frac{r_{2}^{4}}{\omega\left(r_{2}\right) F\left(r_{2}\right)^{2} L\left(r_{1}, r_{2},-z\right)}<\frac{4 m^{2}}{r_{2}^{2}} .
$$

Hence

$$
\begin{aligned}
& -S(\Lambda)=\frac{2}{m^{2}} \int_{0}^{1} z^{2} d z \\
& \cdot \int_{\kappa}^{\Lambda} \frac{r_{2}^{4}}{\omega\left(r_{2}\right) F\left(r_{2}\right)^{2} L\left(r_{1}, r_{2},-z\right)} d r_{2} \\
& \cdot \int_{\mathcal{K}}^{\Lambda} \frac{r_{1}^{4}}{\omega\left(r_{1}\right) F\left(r_{1}\right)^{2} L\left(r_{1}, r_{2}, z\right)} d r_{1}<\frac{2}{3 m^{2}} \\
& \cdot \int_{\kappa}^{\Lambda} \frac{4 m^{2}}{r_{2}^{2}} d r_{2} \int_{\mathcal{K}}^{\Lambda} \frac{4 m^{2}}{r_{1}^{2}} d r_{1}<\frac{32 m^{2}}{3 \kappa^{2}} .
\end{aligned}
$$

Since $S(\Lambda)$ is decreasing and bounded below, it converges as $\Lambda \rightarrow \infty$.
Proof of $\lim _{\Lambda \rightarrow \infty}\left(I_{6}(\Lambda) / \log \Lambda\right)=0$. We redefine $h\left(r_{1}, r_{2}, z\right)$, $J(\Lambda), S(\Lambda, z), S_{1}(\Lambda, z)$, and $S_{2}(\Lambda, z)$ as

$$
\begin{aligned}
& h\left(r_{1}, r_{2}, z\right) \\
& =\frac{r_{1}^{2} r_{2}^{2}}{\omega\left(r_{1}\right) \omega\left(r_{2}\right)}\left(\frac{1}{F\left(r_{1}\right)}+\frac{1}{F\left(r_{2}\right)}\right)^{2} \frac{r_{1}^{2}+r_{2}^{2}}{L\left(r_{1}, r_{2}, z\right)^{3}}, \\
& J(\Lambda)=\int_{-1}^{1} \int_{\mathcal{K}}^{\Lambda} \int_{\mathcal{K}}^{\Lambda} h\left(r_{1}, r_{2}, z\right) d z d r_{2} d r_{1}, \\
& S(\Lambda, z)=\int_{\mathcal{K}}^{\Lambda} \int_{\mathcal{K}}^{\Lambda} h\left(r_{1}, r_{2}, z\right) d r_{1} d r_{2}, \\
& S_{1}(\Lambda, z)=2 \int_{\mathcal{K}}^{\Lambda} \int_{r_{2}}^{2 r_{2}} h\left(r_{1}, r_{2}, z\right) d r_{2} d r_{1}, \\
& S_{2}(\Lambda, z)=2 \int_{\mathcal{K}}^{\Lambda} \int_{2 r_{2}}^{\Lambda} h\left(r_{1}, r_{2}, z\right) d r_{2} d r_{1} .
\end{aligned}
$$

We have $I_{6}(\Lambda)=\left(\pi^{2} /(2 \pi)^{6}\right) J(\Lambda)$.

Step 1. Our first task is to prove that $\lim _{\Lambda \rightarrow \infty} S(\Lambda, z)$ exists for all $z \in I$. Since $h\left(r_{1}, r_{2}, z\right)>0, S(\Lambda, z)$ is increasing in $\Lambda$. We have

$$
S(\Lambda, z)=S_{1}(\Lambda, z)+S_{2}(\Lambda, z)
$$

in the same way as (137). When $r_{2} \leq r_{1}$, it holds that

$$
\begin{array}{r}
r_{1}^{2}+r_{2}^{2} \leq 2 r_{1}^{2}, \\
\frac{1}{F\left(r_{1}\right)}+\frac{1}{F\left(r_{2}\right)}<\frac{4 m}{r_{2}^{2}} .
\end{array}
$$

When $r_{2} \leq r_{1} \leq 2 r_{2}$, it also holds that

$$
\frac{1}{F\left(r_{1}\right)}+\frac{1}{F\left(r_{2}\right)}<\frac{10 m}{r_{1}^{2}}
$$

Then we have

$$
\begin{aligned}
\int_{r_{2}}^{2 r_{2}} h\left(r_{1}, r_{2}, z\right) d r_{1} & <\int_{r_{2}}^{2 r_{2}} \frac{r_{1}^{2} r_{2}^{2}}{r_{1} r_{2}}\left(\frac{10 m}{r_{1}^{2}}\right)^{2} \frac{2 r_{1}^{2}}{r_{1}^{3}} d r_{1} \\
& =\frac{175 m^{2}}{3 r_{2}^{2}} .
\end{aligned}
$$

Hence

$$
S_{1}(\Lambda, z)<\frac{350 m^{2}}{3} \int_{\kappa}^{\Lambda} \frac{d r_{2}}{r_{2}^{2}}<\frac{350 m^{2}}{3 \kappa} .
$$

Let $2 r_{2} \leq r_{1}$. Since $r_{1} / 2 \leq r_{1}-r_{2}$, we have

$$
\frac{r_{1}^{2}}{8 m} \leq \frac{\left(r_{1}-r_{2}\right)^{2}}{2 m}<L\left(r_{1}, r_{2}, z\right) .
$$


Then

$$
\begin{aligned}
& \int_{2 r_{2}}^{\Lambda} h\left(r_{1}, r_{2},\right) d r_{1} \\
& \quad<\int_{2 r_{2}}^{\Lambda} \frac{r_{1}^{2} r_{2}^{2}}{r_{1} r_{2}}\left(\frac{4 m}{r_{2}^{2}}\right)^{2}\left(\frac{8 m}{r_{1}^{2}}\right)^{3} 2 r_{1}^{2} d r_{1} \\
& \quad=\frac{16384 m^{5}}{r_{2}^{3}} \int_{2 r_{2}}^{\Lambda} \frac{d r_{1}}{r_{1}^{3}}<\frac{2048 m^{5}}{r_{2}^{5}} .
\end{aligned}
$$

Therefore

$$
S_{2}(\Lambda, z)<4096 m^{5} \int_{\mathcal{K}}^{\Lambda} \frac{d r_{2}}{r_{2}^{5}}<\frac{1024 m^{5}}{\kappa^{4}} .
$$

From (160), (164), and (167), it follows that $S(\Lambda, z)<$ $350 m^{2} / 3 \kappa+1024 m^{5} / \kappa^{4}$. Since $S(\Lambda, z)$ is increasing in $\Lambda$ and bounded above, it converges as $\Lambda$ goes to infinity.

Step 2. Our second task is to prove $J(\Lambda)$ converges as $\Lambda$ goes to infinity. This step is the same as that of $\lim _{\Lambda \rightarrow \infty}\left(I_{4}(\Lambda) / \log \Lambda\right)=0$.

Proof of $\lim _{\Lambda \rightarrow \infty}\left(I_{7}(\Lambda) / \log \Lambda\right)=0$. We redefine $h\left(r_{1}, r_{2}, z\right)$, $g\left(r_{1}, r_{2}, z\right)$, and $S(\Lambda)$ as

$$
\begin{aligned}
& h\left(r_{1}, r_{2}, z\right) \\
& \quad=\frac{z r_{1}^{3} r_{2}^{3}}{\omega\left(r_{1}\right) \omega\left(r_{2}\right)}\left(\frac{1}{F\left(r_{1}\right)}+\frac{1}{F\left(r_{2}\right)}\right)^{2} \frac{1}{L\left(r_{1}, r_{2}, z\right)^{3}}, \\
& g\left(r_{1}, r_{2}, z\right)=h\left(r_{1}, r_{2}, z\right)+h\left(r_{1}, r_{2},-z\right), \\
& S(\Lambda)=\int_{-1}^{1} \int_{\mathcal{K}}^{\Lambda} \int_{\mathcal{K}}^{\Lambda} h\left(r_{1}, r_{2}, z\right) d z d r_{1} d r_{2} .
\end{aligned}
$$

Then we have $I_{7}(\Lambda)=\left(2 \pi^{2} /(2 \pi)^{6}\right) S(\Lambda)$, and

$$
S(\Lambda)=\int_{0}^{1} \int_{\mathcal{K}}^{\Lambda} \int_{\mathcal{K}}^{\Lambda} g\left(r_{1}, r_{2}, z\right) d z d r_{1} d r_{2}
$$

in the same way as $\lim _{\Lambda \rightarrow \infty}\left(I_{3}(\Lambda) / \log \Lambda\right)=0$. We define $g_{1}\left(r_{1}, r_{2}, z\right)$ and $g_{2}\left(r_{1}, r_{2}, z\right)$ as

$$
\begin{gathered}
g_{1}\left(r_{1}, r_{2}, z\right)=-\frac{6 z^{2} r_{1}^{4} r_{2}^{4}}{m \omega\left(r_{1}\right) \omega\left(r_{2}\right)}\left(\frac{1}{F\left(r_{1}\right)}+\frac{1}{F\left(r_{2}\right)}\right)^{2} \\
. \frac{\left(\left(r_{1}^{2}+r_{2}^{2}\right) / 2 m+\omega\left(r_{1}\right)+\omega\left(r_{2}\right)\right)^{2}}{L\left(r_{1}, r_{2}, z\right)^{3} L\left(r_{1}, r_{2},-z\right)^{3}}, \\
g_{2}\left(r_{1}, r_{2}, z\right) \\
=-\frac{2 z^{4} r_{1}^{6} r_{2}^{6}}{m^{3} \omega\left(r_{1}\right) \omega\left(r_{2}\right)}\left(\frac{1}{F\left(r_{1}\right)}+\frac{1}{F\left(r_{2}\right)}\right)^{2} \\
\cdot \frac{1}{L\left(r_{1}, r_{2}, z\right)^{3} L\left(r_{1}, r_{2},-z\right)^{3}} .
\end{gathered}
$$

Then we have $g\left(r_{1}, r_{2}, z\right)=g_{1}\left(r_{1}, r_{2}, z\right)+g_{2}\left(r_{1}, r_{2}, z\right)$. We redefine $S_{1}(\Lambda)$ and $S_{2}(\Lambda)$ by

$$
\begin{aligned}
& S_{1}(\Lambda)=\int_{0}^{1} \int_{\mathcal{K}}^{\Lambda} \int_{\mathcal{K}}^{\Lambda} g_{1}\left(r_{1}, r_{2}, z\right) d z d r_{1} d r_{2}, \\
& S_{2}(\Lambda)=\int_{0}^{1} \int_{\mathcal{K}}^{\Lambda} \int_{\mathcal{K}}^{\Lambda} g_{2}\left(r_{1}, r_{2}, z\right) d z d r_{1} d r_{2} .
\end{aligned}
$$

Then

$$
S(\Lambda)=S_{1}(\Lambda)+S_{2}(\Lambda) .
$$

Since $g_{1}\left(r_{1}, r_{2}, z\right) \leq 0, S_{1}(\Lambda)$ is decreasing in $\Lambda$. We divide $S_{1}(\Lambda)$ in the following way:

$$
\begin{aligned}
S_{1}(\Lambda)= & \int_{0}^{1} \int_{\mathcal{K}}^{\Lambda} \int_{r_{1}}^{\Lambda} g_{1}\left(r_{1}, r_{2}, z\right) d z d r_{1} d r_{2} \\
& +\int_{0}^{1} \int_{\mathcal{K}}^{\Lambda} \int_{r_{2}}^{\Lambda} g_{1}\left(r_{1}, r_{2}, z\right) d z d r_{2} d r_{1} \\
= & \int_{0}^{1} \int_{\mathcal{K}}^{\Lambda} \int_{r_{1}}^{\Lambda} g_{1}\left(r_{1}, r_{2}, z\right) d z d r_{1} d r_{2} \\
& +\int_{0}^{1} \int_{\mathcal{K}}^{\Lambda} \int_{r_{1}}^{\Lambda} g_{1}\left(r_{2}, r_{1}, z\right) d z d r_{1} d r_{2} \\
= & 2 \int_{0}^{1} \int_{\mathcal{K}}^{\Lambda} \int_{r_{1}}^{\Lambda} g_{1}\left(r_{1}, r_{2}, z\right) d z d r_{1} d r_{2} .
\end{aligned}
$$

Let $\kappa \leq r_{1} \leq r_{2}$. Then we have

$$
\frac{r_{1}^{2}+r_{2}^{2}}{2 m}+\omega\left(r_{1}\right)+\omega\left(r_{2}\right) \leq\left(\frac{1}{m}+\frac{2 \sqrt{\kappa^{2}+v^{2}}}{\kappa^{2}}\right) r_{2}^{2}
$$

in the same way as (124). Let $r_{1} \leq r_{2} \leq 2 r_{1}$. Then we also have

$$
\frac{1}{F\left(r_{1}\right)}+\frac{1}{F\left(r_{2}\right)}<\frac{10 m}{r_{2}^{2}} \text {. }
$$

Therefore

$$
\begin{aligned}
& -g_{1}\left(r_{1}, r_{2}, z\right) \leq \frac{6}{m} \frac{z^{2} r_{1}^{4} r_{2}^{4}}{r_{1} r_{2}}\left(\frac{10 m}{r_{2}^{2}}\right)^{2} \\
& \cdot\left\{\left(\frac{1}{m}+\frac{2 \sqrt{\kappa^{2}+v^{2}}}{\kappa^{2}}\right) r_{2}^{2}\right\}\left(\frac{2 m}{r_{2}^{2}}\right)^{3} \frac{1}{r_{2}^{3}} \\
& =4800 m^{4}\left(\frac{1}{m}+\frac{2 \sqrt{\kappa^{2}+v^{2}}}{\kappa^{2}}\right)^{2} \frac{z^{2} r_{1}^{3}}{r_{2}^{6}}, \\
& -\int_{r_{1}}^{2 r_{1}} g_{1}\left(r_{1}, r_{2}, z\right) d r_{2} \\
& \leq 4800 m^{4}\left(\frac{1}{m}+\frac{2 \sqrt{\kappa^{2}+v^{2}}}{\kappa^{2}}\right)^{2} z^{2} r_{1}^{3} \int_{r_{1}}^{2 r_{1}} \frac{d r_{2}}{r_{2}^{6}} \\
& =930 m^{4}\left(\frac{1}{m}+\frac{2 \sqrt{\kappa^{2}+v^{2}}}{\kappa^{2}}\right)^{2} \frac{z^{2}}{r_{1}^{2}} .
\end{aligned}
$$


Hence

$$
\begin{aligned}
& -2 \int_{0}^{1} \int_{\kappa}^{\Lambda} \int_{r_{1}}^{2 r_{1}} g_{1}\left(r_{1}, r_{2}, z\right) d z d r_{1} d r_{2} \\
& \leq 1860 m^{4}\left(\frac{1}{m}+\frac{2 \sqrt{\kappa^{2}+v^{2}}}{\kappa^{2}}\right)^{2} \int_{0}^{1} z^{2} d z \int_{\kappa}^{\Lambda} \frac{d r_{2}}{r_{2}^{2}} \\
& <\frac{620 m^{4}}{\kappa}\left(\frac{1}{m}+\frac{2 \sqrt{\kappa^{2}+v^{2}}}{\kappa^{2}}\right)^{2} .
\end{aligned}
$$

Let $2 r_{1} \leq r_{2}$. Then we have

$$
\frac{1}{F\left(r_{1}\right)}+\frac{1}{F\left(r_{2}\right)}<\frac{5 m}{2 r_{1}^{2}} .
$$

In addition, since $r_{2} / 2 \leq r_{2}-r_{1}$, we can see that

$$
\frac{r_{2}^{2}}{8 m} \leq \frac{\left(r_{2}-r_{1}\right)^{2}}{2 m}<L\left(r_{1}, r_{2},-z\right) \text {. }
$$

Therefore

$$
\begin{aligned}
- & g_{1}\left(r_{1}, r_{2}, z\right) \leq \frac{6}{m} \frac{z^{2} r_{1}^{4} r_{2}^{4}}{r_{1} r_{2}}\left(\frac{5 m^{2}}{2 r_{1}^{2}}\right)^{2} \\
. & \left\{\left(\frac{1}{m}+\frac{2 \sqrt{\kappa^{2}+v^{2}}}{\kappa^{2}}\right) r_{2}^{2}\right\}^{2}\left(\frac{2 m}{r_{2}^{2}}\right)^{3}\left(\frac{8 m}{r_{2}^{2}}\right)^{3} \\
= & 153600 m^{9}\left(\frac{1}{m}+\frac{2 \sqrt{\kappa^{2}+v^{2}}}{\kappa^{2}}\right)^{2} \frac{z^{2}}{r_{1} r_{2}^{5}} .
\end{aligned}
$$

Then we have

$$
\begin{aligned}
& -\int_{2 r_{1}}^{\Lambda} g_{1}\left(r_{1}, r_{2}, z\right) d r_{2} \\
& \quad \leq \frac{153600 m^{9} z^{2}}{r_{1}}\left(\frac{1}{m}+\frac{2 \sqrt{\kappa^{2}+v^{2}}}{\kappa^{2}}\right)^{2} \int_{2 r_{1}}^{\Lambda} \frac{d r_{2}}{r_{2}^{5}} \\
& \quad \leq 2400 m^{9}\left(\frac{1}{m}+\frac{2 \sqrt{\kappa^{2}+v^{2}}}{\kappa^{2}}\right)^{2} \frac{z^{2}}{r_{1}^{5}} .
\end{aligned}
$$

Hence

$$
\begin{aligned}
& -2 \int_{0}^{1} \int_{\kappa}^{\Lambda} \int_{2 r_{1}}^{\Lambda} g_{1}\left(r_{1}, r_{2}, z\right) d z d r_{1} d r_{2} \\
& \leq 4800 m^{9}\left(\frac{1}{m}+\frac{2 \sqrt{\kappa^{2}+v^{2}}}{\kappa^{2}}\right)^{2} \int_{0}^{1} z^{2} d z \int_{\kappa}^{\Lambda} \frac{d r_{1}}{r_{1}^{5}} \\
& <\frac{400 m^{9}}{\kappa^{4}}\left(\frac{1}{m}+\frac{2 \sqrt{\kappa^{2}+v^{2}}}{\kappa^{2}}\right)^{2} .
\end{aligned}
$$

Then we have

$$
\begin{aligned}
-S_{1}(\Lambda)< & \frac{620 m^{4}}{\kappa}\left(\frac{1}{m}+\frac{2 \sqrt{\kappa^{2}+v^{2}}}{\kappa^{2}}\right)^{2} \\
& +\frac{400 m^{9}}{\kappa^{4}}\left(\frac{1}{m}+\frac{2 \sqrt{\kappa^{2}+v^{2}}}{\kappa^{2}}\right)^{2} .
\end{aligned}
$$

Since $S_{1}(\Lambda)$ is decreasing and bounded below, it converges as $\Lambda \rightarrow \infty$. Since $g_{2}\left(r_{1}, r_{2}, z\right) \leq 0, S_{2}(\Lambda)$ is also decreasing in $\Lambda$. Let $r_{1} \leq r_{2}$. Then

$$
\frac{1}{F\left(r_{1}\right)}+\frac{1}{F\left(r_{2}\right)}<\frac{4 m}{r_{1}^{2}}
$$

Therefore

$$
\begin{aligned}
-g_{2}\left(r_{1}, r_{2}, z\right) & \leq \frac{2}{m^{3}} \frac{z^{4} r_{1}^{6} r_{2}^{6}}{r_{1} r_{2}}\left(\frac{4 m}{r_{1}^{2}}\right)^{2} \frac{8 m^{3}}{r_{2}^{6}} \frac{1}{r_{2}^{3}} \\
& =\frac{256 m^{2} z^{4} r_{1}}{r_{2}^{4}} .
\end{aligned}
$$

Then

$$
\begin{aligned}
& -\int_{r_{1}}^{\Lambda} g_{2}\left(r_{1}, r_{2}, z\right) d r_{2} \leq 256 m^{2} z^{4} r_{1} \int_{r_{1}}^{\Lambda} \frac{d r_{2}}{r_{2}^{4}} \\
& \quad \leq \frac{256 m^{2} z^{4}}{3 r_{1}^{2}} .
\end{aligned}
$$

Hence

$$
-S_{2}(\Lambda) \leq \frac{512 m^{2}}{3} \int_{0}^{1} z^{4} d z \int_{\kappa}^{\Lambda} \frac{d r_{1}}{r_{1}^{2}}<\frac{512 m^{2}}{15 \kappa} .
$$

Since $S_{2}(\Lambda)$ is decreasing in $\Lambda$ and bounded below, it converges. Since both $S_{1}(\Lambda)$ and $S_{2}(\Lambda)$ converge, $S(\Lambda)$ converges.

Proof of $\lim _{\Lambda \rightarrow \infty}\left(I_{8}(\Lambda) / \log \Lambda\right)=0$. We redefine $h\left(r_{1}, r_{2}, z\right)$, $g\left(r_{1}, r_{2}, z\right), g_{1}\left(r_{1}, r_{2}, z\right), g_{2}\left(r_{1}, r_{2}, z\right), S(\Lambda), S_{1}(\Lambda)$, and $S_{2}(\Lambda)$ as

$$
\begin{aligned}
& h\left(r_{1}, r_{2}, z\right)=\frac{z r_{1}^{3} r_{2}^{3}}{\omega\left(r_{1}\right) \omega\left(r_{2}\right)}\left(\frac{1}{F\left(r_{1}\right)}+\frac{1}{F\left(r_{2}\right)}\right) \\
& \frac{1}{L\left(r_{1}, r_{2}, z\right)^{4}}, \\
& g\left(r_{1}, r_{2}, z\right)=h\left(r_{1}, r_{2}, z\right)+h\left(r_{1}, r_{2},-z\right), \\
& g_{1}\left(r_{1}, r_{2}, z\right)=-\frac{2 z^{2} r_{1}^{4} r_{2}^{4}}{m \omega\left(r_{1}\right) \omega\left(r_{2}\right)}\left(\frac{1}{F\left(r_{1}\right)}+\frac{1}{F\left(r_{2}\right)}\right) \\
& \cdot \frac{1}{L\left(r_{1}, r_{2}, z\right) L\left(r_{1}, r_{2},-z\right)^{4}}, \\
& g_{2}\left(r_{1}, r_{2}, z\right)=-\frac{2 z^{2} r_{1}^{4} r_{2}^{4}}{m \omega\left(r_{1}\right) \omega\left(r_{2}\right)}\left(\frac{1}{F\left(r_{1}\right)}+\frac{1}{F\left(r_{2}\right)}\right) \\
& \left.S_{2}(\Lambda)=\int_{\mathcal{K}}^{\Lambda} \int_{r_{2}}^{\Lambda} \int_{1-1 / r_{1}^{1 / 4} r_{2}^{1 / 2}}^{1} g_{1}\left(r_{1}, r_{2}, z\right) d r_{2} d r_{1} d z, z\right), \\
& S_{1}(\Lambda)=\int_{-1}^{1} \int_{\mathcal{K}}^{\Lambda} \int_{\mathcal{K}}^{\Lambda} h\left(r_{1}, r_{2}, z\right) d z d r_{2} d r_{1}, \\
& S_{0}^{1-1 / r_{1}^{1 / 4} r_{2}^{1 / 2}} g_{1}\left(r_{1}, r_{2}, z\right) d r_{2} d r_{1} d z,
\end{aligned}
$$


where

$$
\begin{aligned}
G\left(r_{1}, r_{2}, z\right)= & \frac{1}{L\left(r_{1}, r_{2}, z\right)^{2} L\left(r_{1}, r_{2},-z\right)^{3}} \\
& +\frac{1}{L\left(r_{1}, r_{2}, z\right)^{3} L\left(r_{1}, r_{2},-z\right)^{2}} \\
& +\frac{1}{L\left(r_{1}, r_{2}, z\right)^{4} L\left(r_{1}, r_{2},-z\right)} .
\end{aligned}
$$

Furthermore, we define $S_{3}(\Lambda)$ as

$$
S_{3}(\Lambda)=\int_{0}^{1} \int_{\mathcal{K}}^{\Lambda} \int_{r_{2}}^{\Lambda} g_{2}\left(r_{1}, r_{2}, z\right) d z d r_{2} d r_{1} .
$$

Then we have $I_{8}(\Lambda)=\left(2 \pi^{2} /(2 \pi)^{6}\right) S(\Lambda)$, and

$$
S(\Lambda)=2 \int_{0}^{1} \int_{\kappa}^{\Lambda} \int_{r_{2}}^{\Lambda} g\left(r_{1}, r_{2}, z\right) d z d r_{2} d r_{1}
$$

in the same way as the proof of $\lim _{\Lambda \rightarrow \infty}\left(I_{3}(\Lambda) / \log \Lambda\right)=0$. Since $g\left(r_{1}, r_{2}, z\right)=g_{1}\left(r_{1}, r_{2}, z\right)+g_{2}\left(r_{1}, r_{2}, z\right)$, it holds that

$$
S(\Lambda)=2 S_{1}(\Lambda)+2 S_{2}(\Lambda)+2 S_{3}(\Lambda) .
$$

Since $g_{1}\left(r_{1}, r_{2}, z\right) \leq 0$ and $g_{2}\left(r_{1}, r_{2}, z\right) \leq 0, S_{i}(\Lambda)(i=1,2,3)$ are decreasing in $\Lambda$. Let $r_{2} \leq r_{1}$. Then

$$
\frac{1}{F\left(r_{1}\right)}+\frac{1}{F\left(r_{2}\right)}<\frac{4 m}{r_{2}^{2}}
$$

Let $0 \leq z \leq 1-1 / r_{1}^{1 / 4} r_{2}^{1 / 2}$. Then we have

$$
\frac{1}{(1-z)^{4}} \leq r_{1} r_{2}^{2}
$$

We have

$$
\begin{aligned}
L\left(r_{1}, r_{2},-z\right)= & \frac{\left(r_{1}-r_{2}\right)^{2}+2 r_{1} r_{2}(1-z)}{2 m}+\omega\left(r_{1}\right) \\
& +\omega\left(r_{2}\right)>\frac{r_{1} r_{2}(1-z)}{m} .
\end{aligned}
$$

Using (197) and (198), we have

$$
\frac{1}{L\left(r_{1}, r_{2},-z\right)^{4}}<\frac{m^{4}}{r_{1}^{4} r_{2}^{4}(1-z)^{4}} \leq \frac{m^{4}}{r_{1}^{3} r_{2}^{2}}
$$

From (189), (196), and (199), it follows that

$$
-g_{1}\left(r_{1}, r_{2}, z\right) \leq \frac{2}{m} \frac{z^{2} r_{1}^{4} r_{2}^{4}}{r_{1} r_{2}} \frac{4 m}{r_{2}^{2}} \frac{2 m}{r_{1}^{2}} \frac{m^{4}}{r_{1}^{3} r_{2}^{2}}=\frac{16 m^{5}}{r_{1}^{2} r_{2}} .
$$

Hence we have

$$
\begin{aligned}
-S_{1}(\Lambda) & \leq 16 m^{5} \int_{\mathcal{K}}^{\Lambda} \frac{d r_{2}}{r_{2}} \int_{r_{2}}^{\Lambda} \frac{1}{r_{1}^{2}}\left(1-\frac{1}{r_{1}^{1 / 4} r_{2}^{1 / 2}}\right) d r_{1} \\
& <16 m^{5} \int_{\mathcal{K}}^{\Lambda} \frac{d r_{2}}{r_{2}} \int_{r_{2}}^{\Lambda} \frac{d r_{1}}{r_{1}^{2}}<\frac{16 m^{5}}{\kappa}
\end{aligned}
$$

Since $S_{1}(\Lambda)$ is decreasing in $\Lambda$ and bounded below, it converges. When $r_{2} \leq r_{1}$ and $1-1 / r_{1}^{1 / 4} r_{2}^{1 / 2} \leq z \leq 1$, from (189) and (196), it holds that

$$
-g_{1}\left(r_{1}, r_{2}, z\right)<\frac{2}{m} \frac{r_{1}^{4} r_{2}^{4}}{r_{1} r_{2}} \frac{4 m}{r_{2}^{2}} \frac{2 m}{r_{1}^{2}} \frac{1}{r_{1}^{4}}=\frac{16 m r_{2}}{r_{1}^{3}} .
$$

Hence we have

$$
\begin{aligned}
-S_{2}(\Lambda) & <16 m \int_{\kappa}^{\Lambda} \int_{r_{2}}^{\Lambda} \int_{1-1 / r_{1}^{1 / 4} r_{2}^{1 / 2}}^{1} \frac{r_{2}}{r_{1}^{3}} d r_{2} d r_{1} d z \\
& =16 m \int_{\mathcal{K}}^{\Lambda} \int_{r_{2}}^{\Lambda} \frac{r_{2}}{r_{1}^{3}} r_{1}^{-1 / 4} r_{2}^{-1 / 2} d r_{2} d r_{1} \\
& =16 m \int_{\mathcal{K}}^{\Lambda} r_{2}^{1 / 2} d r_{2} \int_{r_{2}}^{\Lambda} r_{1}^{-13 / 4} d r_{1} \\
& =\frac{64 m}{9} \int_{\mathcal{K}}^{\Lambda} r_{2}^{1 / 2}\left(r_{2}^{-9 / 4}-\Lambda^{-9 / 4}\right) d r_{2} \\
& <\frac{64 m}{9} \int_{\kappa}^{\Lambda} r_{2}^{-7 / 4} d r_{2} \\
& =\frac{256 m}{27}\left(\kappa^{-3 / 4}-\Lambda^{-3 / 4}\right)<\frac{256 m}{27 \kappa^{3 / 4}}
\end{aligned}
$$

Since $S_{2}(\Lambda)$ is decreasing in $\Lambda$ and bounded below, it converges. We have

$$
G\left(r_{1}, r_{2}, z\right)<\frac{4 m^{2}}{r_{1}^{7}}+\frac{8 m^{3}}{r_{1}^{8}}+\frac{16 m^{4}}{r_{1}^{9}}
$$

From (190), (196), and (204), we have

$$
\begin{aligned}
& -g_{2}\left(r_{1}, r_{2}, z\right) \\
& \leq \frac{2}{m} \frac{z^{2} r_{1}^{4} r_{2}^{4}}{r_{1} r_{2}} \frac{4 m}{r_{2}^{2}}\left(\frac{4 m^{2}}{r_{1}^{7}}+\frac{8 m^{3}}{r_{1}^{8}}+\frac{16 m^{4}}{r_{1}^{9}}\right) \\
& <8 r_{1}^{3} r_{2}\left(\frac{4 m^{2}}{r_{1}^{7}}+\frac{8 m^{3}}{r_{1}^{8}}+\frac{16 m^{4}}{r_{1}^{9}}\right) \\
& =\frac{32 m^{2} r_{2}}{r_{1}^{4}}+\frac{64 m^{3} r_{2}}{r_{1}^{5}}+\frac{128 m^{4} r_{2}}{r_{1}^{6}} .
\end{aligned}
$$

Hence

$$
\begin{aligned}
-S_{3}(\Lambda)< & 32 m^{2} \int_{\mathcal{K}}^{\Lambda} r_{2} d r_{2} \int_{r_{2}}^{\Lambda} \frac{d r_{1}}{r_{1}^{4}} \\
& +64 m^{3} \int_{\mathcal{\kappa}}^{\Lambda} r_{2} d r_{2} \int_{r_{2}}^{\Lambda} \frac{d r_{1}}{r_{1}^{5}} \\
& +128 m^{4} \int_{\mathcal{K}}^{\Lambda} r_{2} d r_{2} \int_{r_{2}}^{\Lambda} \frac{d r_{1}}{r_{1}^{6}} \\
< & \frac{32 m^{2}}{3} \int_{\kappa}^{\Lambda} \frac{1}{r_{2}^{2}} d r_{2}+16 m^{3} \int_{\kappa}^{\Lambda} \frac{1}{r_{2}^{3}} d r_{2}
\end{aligned}
$$




$$
\begin{gathered}
+\frac{128 m^{4}}{5} \int_{\kappa}^{\Lambda} \frac{1}{r_{2}^{4}} d r_{2} \\
<\frac{32 m^{2}}{3 \kappa}+\frac{8 m^{3}}{\kappa^{2}}+\frac{128 m^{4}}{15 \kappa^{3}}
\end{gathered}
$$

Since $S_{3}(\Lambda)$ is decreasing in $\Lambda$ and bounded below, it converges. Since $S_{i}(\Lambda)(i=1,2,3)$ converge, $I_{8}(\Lambda)$ converges by (195).

\section{Concluding Remarks}

(1) The Nelson model is defined as the self-adjoint operator

$$
H_{V}=\left(-\frac{1}{2} \Delta+V\right) \otimes 1+1 \otimes H_{f}+\alpha \int_{\mathbb{R}^{3}}^{\oplus} \phi(x) d x,
$$

acting in the Hilbert space $L^{2}(\mathbb{R}) \otimes \mathscr{F} \cong \int_{\mathbb{R}^{3}}^{\oplus} \mathscr{F} d x$. Here $V$ : $\mathbb{R}^{3} \rightarrow \mathbb{R}$ is an external potential and

$$
\begin{aligned}
& \phi(x) \\
& =\frac{1}{\sqrt{2}} \int\left\{\frac{a^{\dagger}(k) e^{-i k x} \hat{\varphi}(k)}{\sqrt{\omega(k)}}+\frac{a(k) e^{i k x} \hat{\varphi}(-k)}{\sqrt{\omega(k)}}\right\} d k .
\end{aligned}
$$

In the case of $V=0, H_{V=0}$ is translation invariant and the relationship between $H_{V}$ and $H(p)$ is given by

$$
H_{V=0}=\int_{\mathbb{R}^{3}}^{\oplus} H(p) d p .
$$

Furthermore the ground state energy of $H(p=0)$ coincides with that of $H_{V=0}$.

(2) We show that $m_{\text {eff }}(\Lambda) / m=1+\sum_{n=1}^{\infty} a_{n}(\Lambda) \alpha^{2 n}$ and $\lim _{\Lambda \rightarrow \infty} a_{2}(\Lambda)= \pm \infty$. It is also expected that $\lim _{\Lambda \rightarrow \infty} a_{n}(\Lambda)$ diverges and the signatures are alternatively changed. Hence $\lim _{\Lambda \rightarrow \infty} m_{\text {eff }}(\Lambda) / m$ may converge but it is not trivial to see it directly.

(3) The relativistic Nelson model is defined by replacing $-(1 / 2) \Delta+V$ with the semirelativistic Schrödinger operator $\sqrt{-\Delta+1}+V$ in (207); that is,

$$
H_{V}^{\mathrm{rel}}=(\sqrt{-\Delta+1}+V) \otimes 1+1 \otimes H_{f}+\int_{\mathbb{R}^{3}}^{\oplus} \phi(x) d x .
$$

Then it follows that

$$
H_{V=0}^{\mathrm{rel}}=\int_{\mathbb{R}^{3}}^{\oplus} H^{\mathrm{rel}}(p) d p,
$$

where $H^{\mathrm{rel}}(p)=\sqrt{\left(p-P_{f}\right)^{2}+1}+H_{f}+\phi(0)$. Then the effective mass $m_{\text {eff }}(\Lambda)$ of $H^{\text {rel }}(p)$ is defined in the same way as that of $H(p)$. We are also interested in seeing the asymptotic behavior of $m_{\text {eff }}(\Lambda)$ as $\Lambda \rightarrow \infty$. However $\sqrt{\left(p-P_{f}\right)^{2}+1}$ is a nonlocal operator and then estimates are rather complicated.

Another interesting nonlocal model is the so-called semirelativistic Pauli-Fierz model defined by

$$
\begin{aligned}
H_{V}^{\mathrm{PF}}= & \sqrt{\left(-i \nabla \otimes-\alpha \int_{\mathbb{R}^{3}}^{\oplus} A(x) d x\right)^{2}+1}+V \otimes 1+1 \\
& \otimes H_{f},
\end{aligned}
$$

where $A(x)$ is a quantized radiation field. See [9] for the detail. Then it follows that

$$
H_{V=0}^{\mathrm{PF}}=\int_{\mathbb{R}^{3}}^{\oplus} H^{\mathrm{PF}}(p) d p,
$$

where $H^{\mathrm{PF}}(p)=\sqrt{\left(p-P_{f}-\alpha A(0)\right)^{2}+1}+H_{f}$. It is also interesting to investigate the asymptotic behavior of the effective mass of the semirelativistic Pauli-Fierz model.

\section{Competing Interests}

The authors declare that there is no conflict of interests regarding the publication of this paper.

\section{Acknowledgments}

Susumu Osawa is grateful to Asao Arai for helpful comments and financial support. This work is financially supported by Grant-in-Aid for Science Research(B) $16 \mathrm{H} 03942$ and Grantin-Aid for challenging Exploratory Research 15K13445 from JSPS.

\section{References}

[1] E. Nelson, "Interaction of nonrelativistic particles with a quantized scalar field," Journal of Mathematical Physics, vol. 5, no. 9, pp. 1190-1197, 1964.

[2] H. Spohn, "Effective mass of the polaron: a functional integral approach," Annals of Physics, vol. 175, no. 2, pp. 278-318, 1987.

[3] F. Hiroshima and H. Spohn, "Mass renormalization in nonrelativistic quantum electrodynamics," Journal of Mathematical Physics, vol. 46, no. 4, Article ID 042302, pp. 42302-42328, 2005.

[4] F. Hiroshima and K. R. Ito, "Effective mass of nonrelativistic quantum electrodynamics," RIMS Kokyuroku, vol. 1492, pp. 2248, 2006.

[5] F. Hiroshima and K. R. Ito, "Mass renormalization in nonrelativistic quantum electrodynamics with spin 1/2," Reviews in Mathematical Physics, vol. 19, no. 4, pp. 405-454, 2007.

[6] V. Bach, T. Chen, J. Fröhlich, and I. M. Sigal, "The renormalized electron mass in non-relativistic quantum electrodynamics," Journal of Functional Analysis, vol. 243, no. 2, pp. 426-535, 2007.

[7] J. Fröhlich and A. Pizzo, "Renormalized electron mass in nonrelativistic QED," Communications in Mathematical Physics, vol. 294, no. 2, pp. 439-470, 2010.

[8] M. Reed and B. Simon, Methods of Modern Mathematical Physics IV, Academic Press, 1978.

[9] F. Hiroshima, "Functional integral approach to semi-relativistic Pauli-Fierz models," Advances in Mathematics, vol. 259, pp. 784840, 2014. 


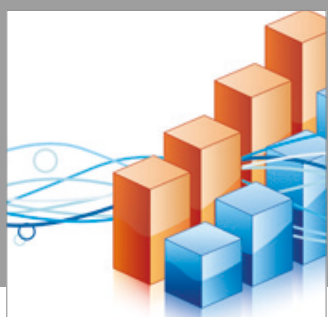

Advances in

Operations Research

vatersals

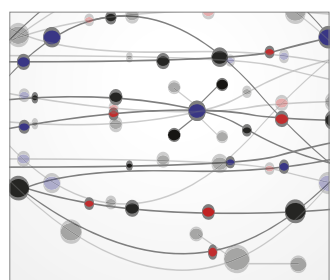

\section{The Scientific} World Journal
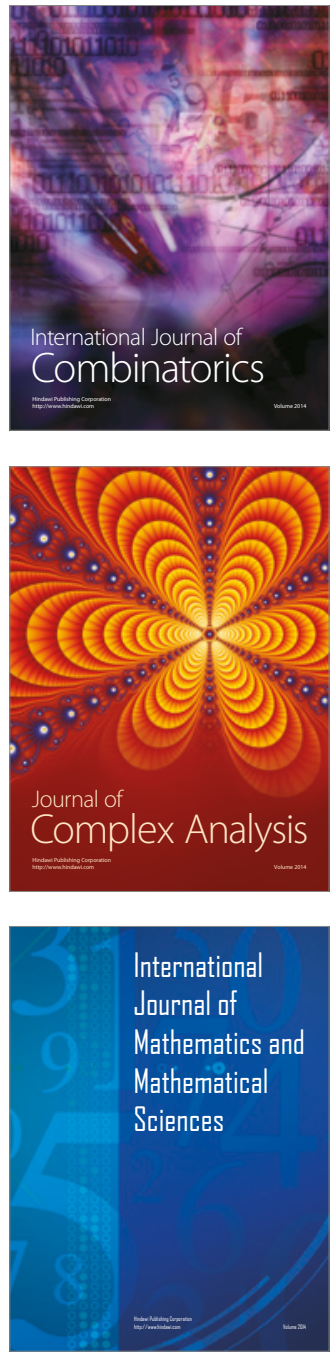
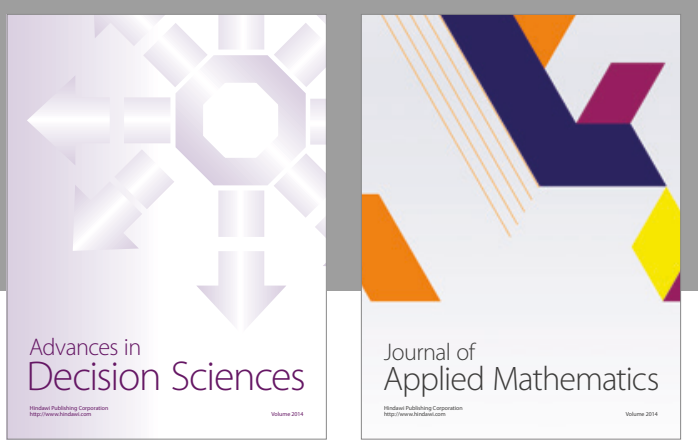

Algebra

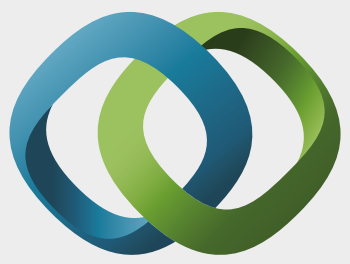

\section{Hindawi}

Submit your manuscripts at

https://www.hindawi.com
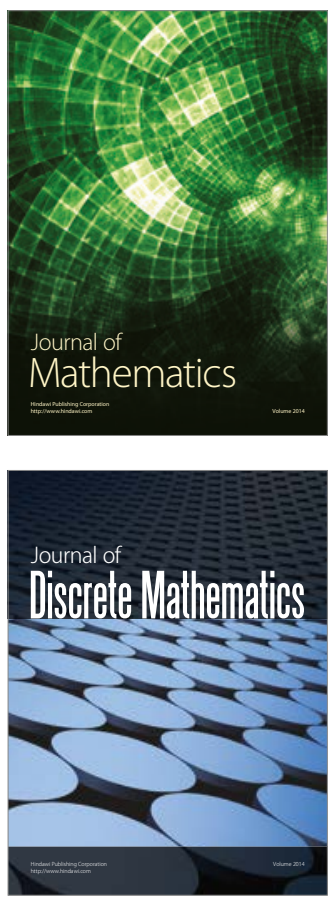

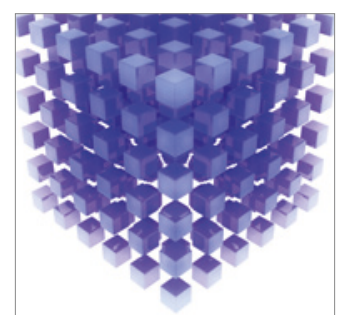

Mathematical Problems in Engineering
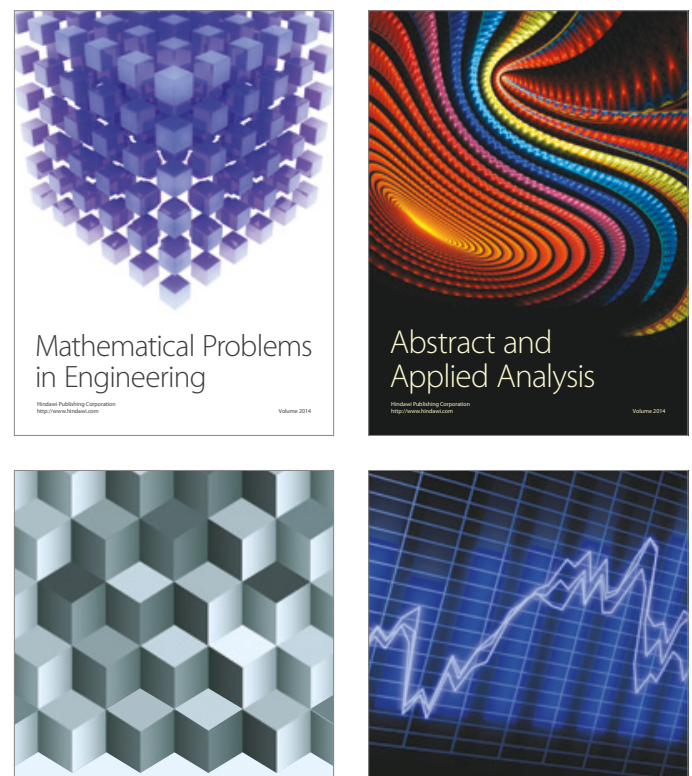

Journal of

Function Spaces

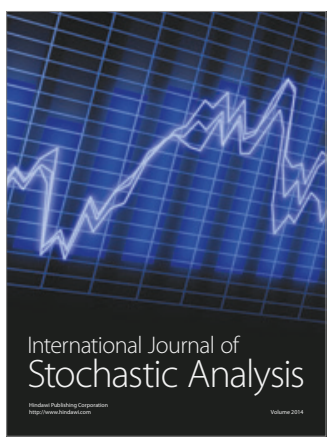

Probability and Statistics
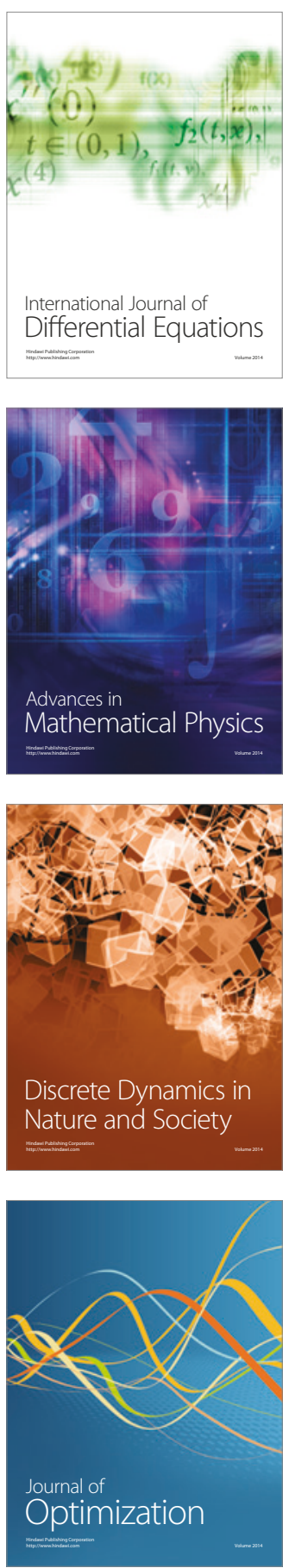